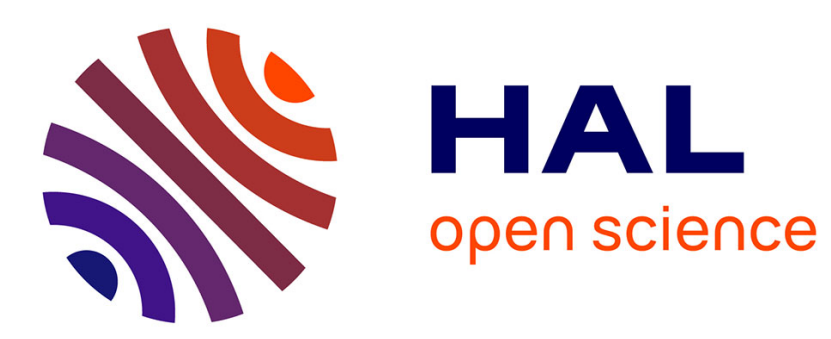

\title{
Integrated Shift Scheduling and Load Assignment Optimization for Attended Home Delivery
}

\author{
Maria Restrepo, Frédéric Semet, Thomas Pocreau
}

\section{To cite this version:}

Maria Restrepo, Frédéric Semet, Thomas Pocreau. Integrated Shift Scheduling and Load Assignment Optimization for Attended Home Delivery. Transportation Science, 2019, 53 (4), pp.917-1212. 10.1287/trsc.2018.0857 . hal-01963916

\section{HAL Id: hal-01963916 https://hal.inria.fr/hal-01963916}

Submitted on 21 Dec 2018

HAL is a multi-disciplinary open access archive for the deposit and dissemination of scientific research documents, whether they are published or not. The documents may come from teaching and research institutions in France or abroad, or from public or private research centers.
L'archive ouverte pluridisciplinaire HAL, est destinée au dépôt et à la diffusion de documents scientifiques de niveau recherche, publiés ou non, émanant des établissements d'enseignement et de recherche français ou étrangers, des laboratoires publics ou privés. 


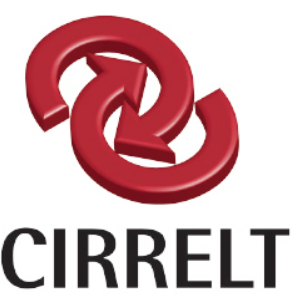

Centre interuniversitaire de recherche sur les réseaux d'entreprise, la logistique et le transport Interuniversity Research Centre

on Enterprise Networks, Logistics and Transportation

\title{
Integrated Shift Scheduling and Load Assignment Optimization for Attended Home Delivery
}

\author{
María I. Restrepo \\ Frédéric Semet \\ Thomas Pocreau
}

June 2017

CIRRELT-2017-32

\footnotetext{
Bureaux de Montréal :

www.cirrelt.ca
} 


\title{
Integrated Shift Scheduling and Load Assignment Optimization for Attended Home Delivery
}

\author{
María I. Restrepo ${ }^{1, *}$, Frédéric Semet ${ }^{2}$, Thomas Pocreau ${ }^{3}$ \\ 1. Interuniversity Research Centre on Enterprise Networks, Logistics and Transportation \\ (CIRRELT), Département de mathématiques et de génie industriel, Polytechnique \\ Montréal, Montréal, Canada, and Inria-Lille, Nord Europe, Lille, France. \\ 2. Ecole Centrale de Lille, CRIStAL, Avenue Paul Langevin, BP 48, 59651 Villeneuve d'Ascq \\ CEDEX, France \\ 3. Colisweb, 165 Avenue de Bretagne, 59000 Lille, France
}

Abstract. In this paper, we study an integrated shift scheduling and load assignment optimization problem for attended home delivery. The proposed approach is divided into two phases, each one corresponding to a different planning level: tactical and operational. In the tactical planning, a daily master plan is generated for each courier. This master plan defines the working shifts, the origin-destination pairs to visit, and the number of client requests to serve. In the operational planning, delivery orders are allocated to couriers in real-time. The stochastic and dynamic nature of client orders is included in the tactical and operational decision levels, respectively. Results on real-world based instances from a delivery company, demonstrate that our approach provides robust tactical solutions that easily accommodate to fluctuations in client orders, preventing additional costs related to the underutilization of couriers and to the use of external couriers to satisfy all delivery requests.

Keywords. Attended home delivery, two stage stochastic programming, L-shaped method, stochastic and dynamic order requirements, robust tactical planning.

Acknowledgements. The authors would like to thank Bpi France, who supported this work with the grant: Concours d'innovation numérique, 2ème édition, 2015-2017.

Results and views expressed in this publication are the sole responsibility of the authors and do not necessarily reflect those of CIRRELT.

Les résultats et opinions contenus dans cette publication ne reflètent pas nécessairement la position du CIRRELT et n'engagent pas sa responsabilité.

\footnotetext{
* Corresponding author: Maria-Isabel.Restrepo@cirrelt.ca

Dépôt légal - Bibliothèque et Archives nationales du Québec Bibliothèque et Archives Canada, 2017

(c) Restrepo, Semet, Pocreau and CIRRELT, 2017
} 


\section{Introduction}

Attended home delivery simplifies and speeds the process of transporting goods by providing convenient, guaranteed and fast on demand movement of packages. This service may be necessary for security reasons (e.g., electronics, expensive computer equipments), because goods are perishable (e.g., groceries) or because goods are physically large (e.g., furniture) (Agatz et al. 2008). The classical model for attended home delivery utilizes couriers who serve client requests through bicycles, motorcycles, cars, and vans and that communicate via mobile phone. Most of the vehicles perform short-duration delivery routes with respect to the planning horizon, due to the characteristics of the package (e.g., perishable goods), the delivery type (e.g., instant delivery), and the vehicle capacity. Each client request consists of a pair of pickup and delivery locations associated with a tight delivery time window. These requests are usually done with little or no notice and their occurrence depends on several factors, as the day of the week and the hour of the day.

Realistic applications of attended home delivery often include the integration of different types of decision planning levels: strategic level, tactical level, and operational level. Strategic planning includes long-term decisions related to the acquisition of vehicles or to the definition of couriers types of contracts. Tactical planning incorporates mid-term decisions associated with the definition of courier schedules or with the choice of delivery time slots to offer to clients. Moreover, operational planning generally includes short-term decisions related to the allocation of delivery requests to the couriers. The inherent connection between these decision levels, the stochastic and dynamic nature of demands, the narrow delivery time windows, and the couriers preferences and availabilities, make the problem of attended home delivery an important logistical challenge, in which these elements must be included in the modeling and solution process of the problem. Otherwise, the resulting plan may lead to unbalanced working schedules, to cost-inefficient delivery routes, and to unfeasible delivery routes that will be unable to satisfy all client requests, since a large portion of them are uncertain.

In this paper, we study an integrated shift scheduling and load assignment problem for attended home delivery under stochastic order requests. In this problem, staffing decisions (allocation of working shifts and geographic zones to couriers), that are feasible to schedule without knowing the actual realization of demand, will be generated in advance (e.g., one day ahead), while short-term adjustments (order allocation to the couriers) are made once real demand information becomes available. The aim of our study is to propose a model for the generation of a robust tactical plan including: i) the design of daily time-space schedules defined by a shift start time, a shift length, and the geographic zones to visit at each working time period; ii) the allocation of couriers to those schedules. This tactical plan will be able to accommodate changes in operational decisions (order allocation to couriers), due to the stochastic variation of order requests. The approach presented in this paper is general enough to be applied to a wide range of attended home delivery problems. However, this study is motivated by operations at Colisweb, a start-up company that offers last-mile delivery services in almost all major cities in France.

Colisweb operates with two types of couriers: dedicated and external. Dedicated couriers are characterized by their availabilities, preferences, and contract types, which are defined by a fixed cost per hour and a variable cost per order delivered. External couriers are always available and do not own a contract, as they are paid based on the number of orders delivered per day. The company offers two types of services: same-day instant order delivery and next-day order delivery for small and light packages. In the former type, packages (orders) must be delivered in the next two hours after the order have been placed. In the latter type, the client must choose between a predefined set of two-hour or three-hour time slots that cover the next two days after the order have been placed (e.g., if a client places an order on Monday morning, he can choose between several delivery time 
slots offered on Tuesday and Wednesday). Client orders are never rejected, since the company can always use an external courier. The territory covered by the company is divided into geographic zones (districts) such as postal codes or neighborhoods. These geographic zones define the different origin destination pairs, denoted as o- $d$ pairs. In addition, couriers utilize different transportation modes, deliveries are served by several depots located within the metropolitan area of the city, and clients are differentiated based on their geographic zone. The problem addressed is stochastic and dynamic, since at the beginning of the day less than $40 \%$ of client orders are known and new clients requests arrive continuously over time, according to a probability distribution.

To the best of our knowledge, this is the first work in the literature to propose an exact approach to address an integrated shift scheduling and load assignment problem for attended home delivery. Therefore, the main contribution of this paper lies in the presentation of a two-stage stochastic programming model, where robust time-space schedules (tactical first-stage decisions) are defined and allocated to the couriers in advance. Moreover, the delivery order allocation to couriers (operational second-stage decisions) is allowed to be adjusted throughout the day, depending on the arrival of new client orders. The paper also proposes, as a secondary contribution, the development and implementation of a multi-cut L-shaped algorithm adapted to our problem, where the second-stage problem decomposes by time periods and by demand scenarios. An extensive computational study on a set of real-world based instances reveals the value of our approach. This study shows that, the design of a robust master plan that incorporates stochastic information about future requests not only help to reduce operating costs, but it can also enhance the couriers' working conditions and the client service level.

The outline of the paper is the following. Section 2 presents the related research on attended home delivery. The definition and formulation of the problems, as well as the solution approaches developed to solve the tactical and operational planning, are presented in Sections 3 and 4, respectively. Computational experiments are discussed in Section 5. Finally, Section 6 presents the concluding remarks and discuss directions for future research.

\section{Related Research}

Although stochastic and dynamic vehicle routing problems (VRP) have been extensively studied in the literature during the last years (see: Pillac et al. (2013) and Ritzinger, Puchinger, and Hartl (2016), for recent reviews on these problems) only recently attended home delivery problems have begun to get some attention in the operations research community. These problems include a large variety of applications as grocery delivery, furniture delivery, and letters and small parcel delivery, where the main challenge is to provide narrow delivery time windows to ensure client satisfaction, while keeping delivery costs under control. In this vein, M.-M., Krishnamurti, and Laporte (2004) study a problem faced by courier companies serving same-day pickup and delivery requests of letters and small packages. A double-horizon based heuristic is proposed to solve the resulting dynamic pickup and delivery problem with time windows. Sungur et al. (2010), solve an attended home delivery problem where clients appear probabilistically and their service times are uncertain. The authors present a scenario-based stochastic programming method with recourse to model the uncertainty in clients, and robust optimization to handle the uncertainty in service times. A courier routing and scheduling problem with stochastic travel times and stochastic demands is addressed in Yan, Lin, and Lai (2013). The authors propose a time-space network model that includes stochastic information about future demands and travel times, to build optimal courier routes and to dynamically adjust them, once requests become known during the service day.

Another related stream of research in attended home delivery focuses on providing anticipatory 
policies for the acceptance or rejection of future delivery requests. The main objective is to maximize the number of clients served, while considering limited transportation capacities. In this line of work, Bent and Van Hentenryck (2004) propose a multiple scenario approach that continuously generates routing plans for different scenarios, including known and stochastic future requests. The decisions made during execution are based on a distinguished plan, selected by a consensus function, that maximizes the number of accepted requests by controlling the time slots offered. Approximate dynamic programming and simulation are used in Ulmer, Mattfeld, and Köster (2014) and Ehmke and Campbell (2014), respectively, to provide anticipatory confirmation policies and to evaluate the impact generated by new client arrivals in the number of future client confirmations. A rollout algorithm (RA) combined with sampling of future requests is presented in Ulmer et al. (2016) to solve a VRP with stochastic clients. The proposed algorithm maximizes the immediate reward and the expected rewards-to-go, by building routing plans and policies for the confirmation or rejection of new requests.

Significant improvements in operational costs in attended home delivery can be obtained through an appropriate design and selection of the time slots to offer at each geographic zone, or with the selection of incentives to influence clients choices of delivery time windows. As an example, Campbell and Savelsbergh (2005) develop a decision support system to maximize the expected profits in egroceries. The decisions are related to the acceptance or rejection of delivery requests, and if a delivery request is accepted, to the selection of its delivery time slot. The authors demonstrate the importance of considering the opportunity costs related to the acceptance of a delivery request in a certain time slot. The time slot schedule design problem (TSSDP), is studied in Hernandez, Gendreau, and Potvin (2014) and Agatz et al. (2011). In the first work, the authors develop two heuristics for the TSSDP in which a periodic VRP with time windows is solved. In the second work, the authors present and compare two modeling and solution alternatives to the problem: a continuous approximation approach and a mathematical programming model. Computational results indicate that solving the TSSDP yields substantial savings over simply offering all available time slots. The use of incentives to influence clients choices of delivery time windows is studied in Campbell and Savelsbergh (2006), Yang et al. (2016) and Klein et al. (2016). Campbell and Savelsbergh (2006), present different insertion heuristics and linear programming models to compute the value of these incentives. Yang et al. (2016), propose dynamic pricing policies based on a multinomial logit client choice model, to determine which and how much incentive (discount or charge) should be offered to the client at each time slot. Klein et al. (2016) present a mixed-integer linear programming formulation, in which delivery costs are anticipated by explicitly incorporating routing constraints, and client behavior is modeled by a general non-parametric rank-based choice model approach. In these works, simulation studies show that the use of incentives schemes can significantly increase the profitability of companies providing attended home delivery.

The literature review reveals that no exact method has been proposed to integrate shift scheduling and order allocation in an attended home delivery environment. Therefore, the distinctive features of this paper consist in two main aspects: 1) because the time slots offered to the clients are fixed in advance, our tactical problem differs from the time slot management problem. More precisely, the aim of the tactical planning level is to generate a robust master plan including two types of decisions: i) the allocation of time-space schedules to couriers; ii) the assignment of an approximate number of packages to deliver at each time period, for each o-d pair, by each courier. The goal of this master plan is to minimize delivery costs, to include courier preferences and availabilities, and to serve as a starting point for subsequent real-time adjustments; 2) because client requests are never rejected, real-time decisions are related to the allocation of packages to the couriers, so that delivery costs are minimized and the master plan is as least as possible modified. Moreover, our problem includes the possibility to consolidate client requests and, on the contrary from some other authors, our approach 
includes same-day instant deliveries. The mathematical formulation and solution approach for the tactical planning are presented in the next Section.

\section{Tactical Planning}

In defining a model for the integrated shift scheduling and load assignment tactical planning problem we assume that: i) the total number of client orders is divided into two parts: a deterministic part corresponding to the next-day delivery requests and a stochastic part corresponding to the same-day delivery requests; ii) same-day order requests arrive in an uncertain way at the beginning of each period of the day; ii) same-day order requests are served within their period of arrival. In a similar way, next-day order requests are served within the first period they become available (the time period including the start of the time window); iii) since consolidation of orders is allowed and likely to occur, the distance traveled per courier per time period only depends on the o-d pairs visited and it is independent on the number of orders served; iv) there is an infinite number of external couriers, hence client requests are never rejected; v) dedicated couriers visit a maximum number of o-d pairs per time period.

The above assumptions allow us to define a tactical problem that integrates two types of decisions: first-stage decisions and second-stage decisions. First-stage decisions are related to the design of a schedule that includes the periods of the day in which each courier must work and the o-d pairs to visit at each time period. Second-stage decisions (recourse actions) consist of the allocation of a number of orders (packages to be delivered) at each time period, at each o-d pair, by each courier (dedicated and external), such that the demand (number of packages to deliver) for each scenario is satisfied. The objective of the integrated shift scheduling and load assignment problem under demand uncertainty is to minimize the sum of the daily staffing cost (fixed cost) plus the expected daily recourse cost (variable cost), as it will be presented in the next Section.

\subsection{A Two-stage Stochastic Programming Model}

Let $P, A, I$, and $C$ define the set of o-d pairs, the set of geographic zones, the set of time periods in the time horizon, and the set of dedicated couriers, respectively. Each o-d pair $p \in P$ has an associated list of neighbors $N_{p}$ : o-d pair $p^{\prime}$ is a neighbor of $p$, if the geographic zone $a \in A$ associated with the destination of $p$ is adjacent to the geographic zone $a \in A$ associated with the origin of $p^{\prime}$. Each courier $c \in C$ admits a feasible set of shifts represented by $S^{c}$. Shifts $s \in S^{c}, c \in C$ are pre-generated according to the preferences of the courier, its availabilities during the time horizon, and some workplace agreements (e.g., minimum and maximum number of working hours per day and position and length of breaks).

We assume that at the moment we can act on second-stage variables, the scenario for each time period $i \in I$ is fully known. Hence, the allocation of daily shifts and o-d pairs to couriers will be generated in advance, while adjustments (allocation of packages to dedicated couriers and the use of an external courier) are made once real demand information is available. We also make two assumptions related to the stochastic client orders. First, we assume that the random vector, $\xi_{i}$, representing the stochastic value of same-day client orders at each time period $i \in I$ has a finite support. Henceforth, we define $\Omega_{i}$ as the set of its possible realizations and $p_{i}^{(w)}>0$ as the probability of occurrence of scenario $w \in \Omega_{i}$, with $\sum_{w \in \Omega_{i}} p_{i}^{(w)}=1, i \in I$. Second, we assume that the geographic distribution of client orders (i.e., the origin and destination zones associated to each order) is done according to values $p_{a o}, p_{a d}$. These values represent, for each geographic zone $a \in A$, the probability of being selected as an origin and the probability of being selected as a destination, respectively. The parameters used in the formulation of the problem are presented in Table 1 . 


\section{Parameters:}

$f_{s}^{c}: \quad$ Cost associated to shift $s \in S^{c}$ from courier $c \in C$ (fixed cost);

$\delta_{i s}^{c}$ : $\quad$ Parameter that takes value 1 if time period $i \in I$ is a working period in shift $s \in S^{c}$ for courier $c \in C$, and assumes value 0 otherwise;

$\alpha_{i}^{c}$ : $\quad$ Maximum number of o-d pairs that courier $c \in C$ can visit at time period $i \in I$;

$\sigma_{i p}^{c}$ : Average time traveled by courier $c \in C$ when o-d pair $p \in P$ is assigned at time period $i \in I$;

$\tau_{i}^{c}: \quad$ Maximum travel time for courier $c \in C$ at time period $i$;

$l_{i p}^{c}: \quad$ Non-negative cost associated to the delivery of one package by courier $c \in C$, at time period $i \in I$, for o-d pair $p \in P$ (variable cost);

$c_{i p}$ : Non-negative cost associated to the delivery of one package by an external courier at time period $i$, for o-d pair $p, c_{i p}>l_{i p}^{c}, i \in I, p \in P, c \in C$;

$\beta_{i}^{c}$ : $\quad$ Maximum number of packages to deliver at time period $i \in I$ by courier $c \in C$;

$\beta_{i p}^{c}: \quad$ Maximum number of packages to deliver at time period $i \in I$, for o-d pair $p \in P$ by courier $c \in C, \beta_{i p}^{c} \leq \beta_{i}^{c}, c \in C, i \in I, p \in P$;

$n d_{i p}: \quad$ Number of next-day packages to deliver for o-d pair $p \in P$, at time period $i \in I$;

$s d_{i p}$ : Mean of the number of same-day packages to deliver for o-d pair $p \in P$, at time period $i \in I$;

$d_{i p}$ : $\quad$ Mean of the number of packages to deliver for o-d pair $p \in P$, at time period $i \in I$, $d_{i p}=n d_{i p}+s d_{i p}$

$s d_{i p}^{(w)}$ : Number of same-day packages to deliver in o-d pair $p \in P$, at time period $i \in I$, under scenario $w \in \Omega_{i}$;

$d_{i p}^{(w)}: \quad$ Number of packages to deliver in o-d pair $p \in P$, at time period $i \in I$, under scenario $w \in \Omega_{i}, d_{i p}^{(w)}=n d_{i p}+s d_{i p}^{(w)}$

$\mu_{i p}^{c(w)}$ : Minimum value between the demand and the total capacity for each $c \in C, p \in P, i \in$ $I, w \in \Omega_{i}, \mu_{i p}^{c(w)}=\min \left\{d_{i p}^{(w)}, \beta_{i p}^{c}\right\}$.

Table 1 - Notation for the two-stage stochastic programming model.

First-stage variables are represented by $x_{s}^{c}$ and $y_{i p}^{c}$. Variable $x_{s}^{c}$ takes value 1 if courier $c \in C$ is assigned to shift $s \in S^{c}$, and assumes value 0 otherwise. Variable $y_{i p}^{c}$ takes value 1 if courier $c \in C$ covers o-d pair $p \in P$ at time period $i \in I$, and assumes value 0 otherwise. To guarantee feasible courier transitions between o-d pairs among consecutive time periods, we define a set of auxiliary variables $z_{i p}^{c}$ that take value 0 if courier $c \in C$ does not cover o-d pair $p \in P$ at time period $i \in I$, and assumes values 0 or 1 otherwise. The formulation of the two-stage stochastic programming model, denoted as $S S L A$, is as follows: 


$$
\begin{aligned}
(S S L A) \min & \sum_{c \in C} \sum_{s \in S^{c}} f_{s}^{c} x_{s}^{c}+\mathcal{Q}(\mathbf{x}, \mathbf{y}) \\
& \sum_{s \in S^{c}} x_{s}^{c} \leq 1, \forall c \in C, \\
& \sum_{s \in S^{c}} \delta_{i s}^{c} x_{s}^{c} \leq \sum_{p \in P} y_{i p}^{c} \leq \alpha_{i}^{c} \sum_{s \in S^{c}} \delta_{i s}^{c} x_{s}^{c}, \forall c \in C, i \in I, \\
& \sum_{p \in P} \sigma_{i p}^{c} y_{i p}^{c} \leq \tau_{i}^{c} \sum_{s \in S^{c}} \delta_{i s}^{c} x_{s}^{c}, \forall c \in C, i \in I, \\
& \sum_{p^{\prime} \notin N_{p}} y_{i p^{\prime}}^{c} \leq 1-y_{i p}^{c}, \forall c \in C, i \in I, p \in P, \\
& z_{i p}^{c} \leq y_{i p}^{c}, \forall c \in C, i \in I, p \in P, \\
& \sum_{p \in P} z_{i p}^{c} \geq \sum_{s \in S^{c}} \delta_{i s}^{c} x_{s}^{c}, \forall c \in C, i \in I, \\
& z_{i p}^{c}+\sum_{s \in S^{c}} \delta_{i+1 s}^{c} x_{s}^{c} \leq \sum_{p^{\prime} \in N_{p}} y_{i+1 p^{\prime}}^{c}+1, \forall c \in C, i \in I, p \in P, \\
& x_{s}^{c} \in\{0,1\}, \forall c \in C, s \in S^{c}, \\
& y_{i p}^{c} \in\{0,1\}, \forall c \in C, i \in I, p \in P, \\
& z_{i p}^{c} \in\{0,1\}, \forall c \in C, i \in I, p \in P .
\end{aligned}
$$

The objective (1) is to minimize the shift assignment cost, plus the expected delivery cost $\mathcal{Q}(\mathbf{x}, \mathbf{y})$. Constraints (2) correspond to the assignment of at most one shift $s$ to each courier $c$. Constraints (3) set the link between shift variables $x_{s}^{c}$ with o-d pair allocation variables $y_{i p}^{c}$. These constraints also set the minimum and maximum number of o-d pairs $p$ to visit by each courier $c$, at each time period $i$. Constraints (4) limit the time traveled by each courier $c$ at time period $i$. Constraints (5) guarantee that all $\mathrm{o}-\mathrm{d}$ pairs visited by each courier $c$ at each time period $i$ are neighbors. In a similar manner, constraints (6)-(8) define valid o-d pair "transitions" between consecutive time periods. Specifically, if courier $c$ visits a number of o-d pairs in time period $i$ and if $c$ is scheduled to work in the next time period $(i+1), c$ must visit, at $i+1$, at least one of the neighbors of the o-d pairs covered at $i$.

The expected recourse function is denoted by $\mathcal{Q}(\mathbf{x}, \mathbf{y})$, the recourse function at time period $i$ is represented by $\mathcal{Q}\left(\mathbf{x}, \mathbf{y}_{i}, \xi_{i}\right)$. Since random vectors $\xi_{i}, i \in I$ are independent, have finite support, and $\sum_{i \in I} \sum_{w \in \Omega_{i}} p_{i}^{(w)}=1, \mathcal{Q}(\mathbf{x}, \mathbf{y})$ is equivalent to:

$$
\mathcal{Q}(\mathbf{x}, \mathbf{y}) \equiv \mathbb{E}_{\xi}[\mathcal{Q}(\mathbf{x}, \mathbf{y}, \xi)] \equiv \sum_{i \in I} \mathbb{E}_{\xi}\left[\mathcal{Q}\left(\mathbf{x}, y_{i}, \xi_{i}\right)\right] \equiv \sum_{i \in I} \sum_{w \in \Omega_{i}} p_{i}^{(w)} \mathcal{Q}\left(\mathbf{x}, \mathbf{y}_{i}, \xi_{i}(w)\right)
$$

Second-stage variables are denoted by $v_{i p}^{c(w)}$ and $e_{i p}^{(w)}$. These variables represent, respectively, the number of packages delivered by courier $c$ at time period $i$, for o-d pair $p$, under scenario $w$, and the number of packages delivered by an external courier at time period $i$, for o-d pair $p$, under scenario $w$. The formulation of the recourse function $\mathcal{Q}\left(\mathbf{x}, \mathbf{y}_{i}, \xi_{i}(w)\right)$ is as follows: 


$$
\begin{aligned}
\mathcal{Q}\left(\mathbf{x}, \mathbf{y}_{i}, \xi_{i}(w)\right)=\min & \sum_{c \in C} \sum_{p \in P} l_{i p}^{c} v_{i p}^{c(w)}+\sum_{p \in P} c_{i p} e_{i p}^{(w)} \\
& v_{i p}^{c(w)} \leq \mu_{i p}^{c(w)} y_{i p}^{c}, \forall c \in C, p \in P, \\
& \sum_{p \in P} v_{i p}^{c(w)} \leq \beta_{i}^{c} \sum_{s \in S^{c}} \delta_{i s}^{c} x_{s}^{c}, \forall c \in C, \\
& \sum_{c \in C} v_{i p}^{c(w)}+e_{i p}^{(w)}=d_{i p}^{(w)}, \forall p \in P, \\
& v_{i p}^{c(w)} \geq 0, \forall c \in C, p \in P, \\
& e_{i p}^{(w)} \geq 0, \forall p \in P .
\end{aligned}
$$

Recourse function $\mathcal{Q}\left(\mathbf{x}, \mathbf{y}_{i}, \xi_{i}(w)\right)$ corresponds to the minimization of the total delivery cost at time period $i$, under scenario $w$. Constraints (14) guarantee that the number of packages transported by courier $c$ does not exceed its maximum capacity for each o-d pair $p$. Constraints (15) limit the total number of packages assigned to courier $c$ to a maximum capacity. Constraints (16) ensure the demand satisfaction for each o-d pair $p$ for scenario $w$.

Integrality constraints on second-stage variables $v_{i p}^{c(w)}, e_{i p}^{(w)}$ are relaxed since problem 1318 can be stated as a minimum cost flow problem defined on a directed acyclic graph $G_{i}^{(w)}=$ $\left(\mathcal{N}_{i}^{(w)}, \mathcal{A}_{i}^{(w)}\right)$, where $\mathcal{N}_{i}^{(w)}$ is the node set and $\mathcal{A}_{i}^{(w)}$ is the arc set. Set $\mathcal{N}_{i}^{(w)}$ contains a node for each courier $c \in C$, a node that represents the external courier, a node for each o-d pair $p \in P$, and two additional nodes representing the source and sink nodes. These nodes are denoted by $n_{c^{\prime}}, n_{p}, n_{s}$, and $n_{f}$, respectively. Set $\mathcal{A}_{i}^{(w)}$ contains three types of arcs: $\mathcal{A}_{1 i}^{(w)}$ includes the arcs connecting the source node $n_{s}$ with courier nodes (dedicated or external) $n_{c^{\prime}} ; \mathcal{A}_{2 i}^{(w)}$ contains the arcs connecting courier nodes $n_{c^{\prime}}$ with o-d pair nodes $n_{p}$; and $\mathcal{A}_{3 i}^{(w)}$ defines the arcs connecting o-d pair nodes $n_{p}$ with sink node $n_{s}$. An attribute $b(n)$ representing a supply or demand is associated to each node $n$ in $\mathcal{N}_{i}^{(w)}$. Hence $b(n)=\sum_{p \in P} d_{i p}^{(w)}$ if $n=n_{s} ; b(n)=0$ if $n=n_{c}^{\prime} ; b(n)=-d_{i p}^{(w)}$ if $n=n_{p} ;$ and $b(n)=0$ if $n=n_{f}$. To each arc $(n, l) \in \mathcal{N}_{i}^{(w)}$ there are two attributes associated: $c_{n l}$ and $u_{n l}$, representing the cost per unit of flow and the maximum capacity of $\operatorname{arc}(n, l)$, respectively. If $(n, l) \in \mathcal{A}_{1 i}^{(w)}$, $c_{n l}=0$ and $u_{n l}=\beta_{i}^{c} \sum_{s \in S^{c}} \delta_{i s}^{c} x_{s}^{c}$ if $l$ is a node representing a dedicated courier, otherwise $c_{n l}=0$ and $u_{n l}=\sum_{p \in P} d_{i p}^{(w)}\left(l\right.$ represents an external courier). If $(n, l) \in \mathcal{A}_{2 i}^{(w)}, c_{n l}=l_{i p}^{c}$ and $u_{n l}=\mu_{i p}^{c} y_{i p}^{c}$ if $n$ is a node representing a dedicated courier, otherwise $c_{n l}=c_{i p}$ and $u_{n l}=\sum_{p \in P} d_{i p}^{(w)}$ (n represents an external courier). If $(n, l) \in \mathcal{A}_{3 i}^{(w)}, c_{n l}=0$ and $u_{n l}=d_{i p}^{(w)}$. Since all supply values, demand values, and all upper bounds on arc flows are integer, and since the node-arc incidence matrix of a minimum cost flow problem is totally unimodular, $\mathcal{Q}\left(\mathbf{x}, \mathbf{y}_{i}, \xi_{i}(w)\right)$ possesses the integrality property and integrality constraints on second-stage variables $v_{i p}^{c(w)}, e_{i p}^{(w)}$ can be relaxed.

Proposition 1 The two-stage stochastic programming problem (1)-(7) has complete recourse: Proof: Observe that for any realization of the random vector $\xi_{i}, i \in I$ and value of first-stage variables $y_{i p}^{c}, x_{s}^{c}$, problem (13)-(18) is always feasible, as it is always possible to call external couriers with infinite capacity to satisfy the uncovered demand. Specifically, there always exists a feasible flow in $G_{i}^{(w)}=\left(\mathcal{N}_{i}^{(w)}, \mathcal{A}_{i}^{(w)}\right)$ since $u_{n_{s} l}=\sum_{p \in P} d_{i p}^{(w)}$ and $u_{l n}=d_{i p}^{(w)}, \forall n \in P$, with l representing the external courier. 
In the following, we present the solution method to solve the tactical decision level of the integrated shift scheduling and load assignment problem.

\subsection{Multi-cut Integer L-shaped Method}

The L-shaped method was first proposed by Van Slyke and Wets (1969) for solving two-stage stochastic linear problems. The idea behind this method is to approximate the nonlinear term, $\mathcal{Q}(\mathbf{x}, \mathbf{y})$, in the objective function of the two-stage stochastic programming problem (1)-(11). Since the expected recourse function involves solving all second-stage recourse problems, the main principle of the L-shaped method is to avoid numerous function evaluations by using an outer linearization of $\mathcal{Q}(\mathbf{x}, \mathbf{y})$, as in Benders decomposition.

Two types of cuts are sequentially generated in the L-shaped method: feasibility and optimality cuts. While feasibility cuts enforce necessary conditions for feasibility of the primal second stage problem $\{(\mathbf{x}, \mathbf{y}) \mid \mathcal{Q}(\mathbf{x}, \mathbf{y})<+\infty\}$, optimality cuts approximate the term $\mathcal{Q}(\mathbf{x}, \mathbf{y})$ in the objective function of the two-stage stochastic programming problem. Since problem (1)-(11) has complete recourse (Proposition 1), there is no need to generate feasibility cuts. Let $\pi_{i p}^{c(w)}, \phi_{i}^{c(w)}$, and $\lambda_{i p}^{(w)}$ be the dual variables associated with constraints 14, , 15 , and $\sqrt{16}$, respectively. Let $\boldsymbol{\Delta}_{i}^{(w)}$ be the projection over the space of variables $\pi_{i p}^{c(w)}, \phi_{i}^{c(w)}, \lambda_{i p}^{(w)}$ of the polyhedron defined by the constraints associated with the dual of model $\mathcal{Q}\left(\mathbf{x}, \mathbf{y}_{i}, \xi_{i}(w)\right)$. Note that $\boldsymbol{\Delta}_{i}^{(w)}$ is itself a polyhedron (Wolsey and Nemhauser 1999). Let $E_{\boldsymbol{\Delta}_{i}^{(w)}}$ be the set of extreme points of $\boldsymbol{\Delta}_{i}^{(w)}$. By defining $\theta_{i}^{(w)}$ as an additional set of non-negative variables (as the optimal value of second-stage problems is always non-negative), the two-stage stochastic problem $S S L A$ can be reformulated as the following model, denoted as RSLA. 


$$
\begin{aligned}
(R S L A) \min & \sum_{c \in C} \sum_{s \in S^{c}} f_{s}^{c} x_{s}^{c}+\sum_{i \in I} \sum_{w \in \Omega_{i}} \theta_{i}^{(w)} \\
& \sum_{s \in S^{c}} x_{s}^{c} \leq 1, \forall c \in C, \\
& \sum_{s \in S^{c}} \delta_{i s}^{c} x_{s}^{c} \leq \sum_{p \in P} y_{i p}^{c} \leq \alpha_{i}^{c} \sum_{s \in S^{c}} \delta_{i s}^{c} x_{s}^{c}, \forall c \in C, i \in I, \\
& \sum_{p \in P} \sigma_{i p}^{c} y_{i p}^{c} \leq \tau^{c} \sum_{s \in S^{c}} \delta_{i s}^{c} x_{s}^{c}, \forall c \in C, i \in I, \\
& \theta_{i}^{(w)} \geq p_{i}^{(w)}\left(\sum_{c \in C} \sum_{p \in P} \mu_{i p}^{c} \pi_{i p}^{c(w)} y_{i p}^{c}+\sum_{c \in C} \beta_{i}^{c} \phi_{i}^{c(w)} \sum_{s \in S^{c}} \delta_{i s}^{c} x_{s}^{c}+\sum_{p \in P} d_{i p}^{(w)} \lambda_{i p}^{(w)}\right), \\
& \sum_{p^{\prime} \notin N_{p}} y_{i p^{\prime}}^{c} \leq 1-y_{i p}^{c}, \forall c \in C, i \in I, p \in P, \\
& z_{i p}^{c} \leq y_{i p}^{c}, \forall c \in C, i \in I, p \in P, \\
& \sum_{p \in P} z_{i p}^{c} \geq \sum_{s \in S^{c}} \delta_{i s}^{c} x_{s}^{c}, \forall c \in C, i \in I, \\
& \left.z_{i p}^{c}+\sum_{s \in S_{i}^{c}}^{(w)}, \phi_{i}^{(w)}, \lambda_{i}^{(w)}\right) \in E_{s} x_{s}^{c} \leq \sum_{p^{\prime} \in N_{p}} y_{i+1 p^{\prime}}^{c}+1, \forall c \in C, i \in I, p \in P, \\
& x_{s}^{c} \in\{0,1\}, \forall c \in C, s \in S^{c}, \\
& y_{i p}^{c} \in\{0,1\}, \forall c \in C, i \in I, p \in P, \\
& z_{i p}^{c} \in\{0,1\}, \forall c \in C, i \in I, p \in P, \\
& \theta_{i}^{(w)} \geq 0, \forall i \in I, w \in \Omega_{i} .
\end{aligned}
$$

Optimality cuts 23, ensure that the value of each variable $\theta_{i}^{(w)}$ is larger than or equal to the optimal value of its corresponding second-stage problem for each time period $i \in I$ and each scenario $w \in \Omega_{i}$. The structure of problem (19)-31 allows the L-shaped method to be extended to include multiple cuts at each iteration, i.e. one per time period and per scenario, instead of adding one aggregated cut. Birge and Louveaux (1988) showed that in an iterative algorithm, adding multiple cuts at the same iteration may speed up convergence and reduce the number of iterations. Optimality cuts (23) do not need to be exhaustively generated, since only a subset of them are active in the optimal solution of the problem. Hence, an iterative algorithm, with $k$ as the iteration counter, is used to generate only the subset of optimality cuts, $E_{\boldsymbol{\Delta}_{i}^{(w)}}^{k} \subseteq E_{\boldsymbol{\Delta}_{i}^{(w)}}$, (extreme points of $\boldsymbol{\Delta}_{i}^{(w)}$ ) that will represent the optimal solution.

The algorithm used to solve the problem is based on the integer L-shaped method presented in Laporte and Louveaux (1993). This branch-and-cut algorithm (also called one-tree algorithm) operates on a so-called current problem $(\mathrm{CP})$ at each node of the search tree. CP is initially defined by relaxing $R S L A$ in two ways: i) integrality constraints (28)-(30) are relaxed; ii) since the initial set of optimality cuts $E_{\boldsymbol{\Delta}_{i}^{(w)}}^{k}$ is empty, $\mathcal{Q}(\mathbf{x}, \mathbf{y})$ is replaced by a lower bound on the objective function, in our case zero. As in standard branch-and-cut methods, CP is dynamically modified by gradually introducing integrality conditions through the branching process, and by adding optimality cuts (23) 
into CP at integer solutions. This algorithm is enhanced with the strategy presented in McDaniel and Devine (1977), which consists in adding a number of valid optimality cuts to CP before starting the branching process, i.e. at the root node of the branch-and-bound tree. When some criterion is met, i.e. the relative gap between the upper bound and the lower bound of the problem is smaller than a certain value, the method then starts introducing integrality conditions and adding optimality cuts into $\mathrm{CP}$ at integer solutions.

Let $\epsilon_{1}$ be the tolerance parameter we used to stop the generation of relaxed cuts (stop the McDaniel and Devine (1977) strategy). Let $\mathbf{f}$ be the vector of costs associated to the working shifts. Let $\left(\mathbf{x}_{k}^{*}, \mathbf{y}_{k}^{*}, \theta_{k}^{*}\right)$ be an optimal primal solution, continuous or integer, of CP at iteration $k$. Let $\left(\pi_{k}^{*}, \phi_{k}^{*}, \lambda_{k}^{*}\right)$ and $\mathcal{Q}_{k}^{*}\left(\mathbf{x}, \mathbf{y}_{i}, \xi_{i}(w)\right)$ represent an optimal dual solution and the optimal objective value of the second-stage problems $(13)-(18)$ at iteration $k$. Let $\underline{Q}_{k}, \bar{Q}_{k}$ denote a lower bound and an upper bound for the value of the expected recourse function at iteration $k$, respectively. $\underline{Q_{k}}=\sum_{i \in I} \sum_{w \in \Omega_{i}} \theta_{i k}^{(w) *}, \bar{Q}_{k}=\sum_{i \in I} \sum_{w \in \Omega_{i}} p_{i}^{(w)} \mathcal{Q}_{k}^{*}\left(\mathbf{x}, \mathbf{y}_{i}, \xi_{i}(w)\right)$. Let $\bar{z}$ be the global upper bound for the problem. The description of the multi-cut integer L-shaped algorithm is as follows.

Step 1. Set $k:=0, \bar{z}:=0, \theta_{i k}^{(w)} \geq 0, E_{\Delta_{i}^{(w)}}^{k}:=\emptyset, \forall i \in I, w \in \Omega_{i}$. Solve the LP relaxation of problem (19)- 31 .

Step 2. Get the optimal primal solution $\left(\mathbf{x}_{k}^{*}, \mathbf{y}_{k}^{*}, \theta_{k}^{*}\right)$.

Step 3. Solve second-stage problems (13)-18) using $\left(\mathbf{x}_{k}^{*}, \mathbf{y}_{k}^{*}\right)$; get dual solutions $\left(\pi_{k}^{*}, \phi_{k}^{*}, \lambda_{k}^{*}\right)$ and the optimal value of second-stage problems $\mathcal{Q}_{k}^{*}\left(\mathbf{x}, \mathbf{y}_{i}, \xi_{i}(w)\right)$.

Step 4. For each $i \in I, w \in \Omega_{i}$, if $\left(\mathcal{Q}_{k}^{*}\left(\mathbf{x}, \mathbf{y}_{i}, \xi_{i}(w)\right)-\theta_{i k}^{(w) *}\right) / \mathcal{Q}_{k}^{*}\left(\mathbf{x}, \mathbf{y}_{i}, \xi_{i}(w)\right)>0$ add optimality cuts 23) to the current problem (CP).

Step 5. Solve the LP relaxation of problem (19)- $(31)$.

Step 6. Set $k:=k+1$; compute $\underline{Q}_{k}$, $\bar{Q}_{k}$. If $\left(\bar{Q}_{k}-\underline{Q}_{k}\right) / \bar{Q}_{k} \leq \epsilon_{1}$, continue with Step 7 . Otherwise, return to Step 2.

Step 7. The only node in the branch and bound tree corresponds to the current node, i.e. the root-noDen.

Step 8. Select a node from the list, if none exists stop.

Step 9. Set $k:=k+1$ and solve the relaxation of CP; get the optimal primal solution $\left(\mathbf{x}_{k}^{*}, \mathbf{y}_{k}^{*}, \theta_{k}^{*}\right)$.

Step 10. If solution $\left(\mathbf{x}_{k}^{*}, \mathbf{y}_{k}^{*}\right)$ is not integer, branch on a fractional variable and go to Step 8 .

Step 11. Compute $\bar{Q}_{k}$, if $\left(\mathbf{f}^{T} \mathbf{x}_{k}^{*}+\bar{Q}_{k}\right)<\bar{z}$, update $\bar{z}$.

Step 12. If $\theta_{i k}^{(w) *} \geq \mathcal{Q}_{k}^{*}\left(\mathbf{x}, \mathbf{y}_{i}, \xi_{i}(w)\right), \forall i \in I, w \in \Omega_{i}$, fathom the current node and go to Step 8. Otherwise, add optimality cuts 23 to CP, for all $i \in I, w \in \Omega_{i}$ such that $\theta_{i k}^{(w)}<$ $\mathcal{Q}_{k}\left(\mathbf{x}, \mathbf{y}_{i}, \xi_{i}(w)\right), \forall i \in I, w \in \Omega_{i}$ and go to Step 8 .

An optimal tactical solution $\left(\mathbf{x}^{c *}, \mathbf{y}^{c *}, \mathbf{v}^{c(w) *}\right)$ with an objective function value of $\bar{z}$, is obtained after solving the multi-cut L-shaped method. This solution is used to compute the value of the stochastic solution (VSS). The VSS is a standard measure that indicates the expected gain from solving a stochastic model rather than its deterministic counterpart, the expected value problem 
$(\mathrm{EV})$. The value of the stochastic solution is defined as $V S S=E E V-R P$, where $R P=\bar{z}$ corresponds to the optimal value of the two-stage stochastic programming problem (1)-(11) and $E E V$ corresponds to the expected value of using the EV solution. EV is problem (1)-(11) evaluated using the mean scenario $\bar{\xi}_{i}$ for each time period $i \in I$. Given an EV solution $\left(\overline{\mathbf{x}}^{*}, \overline{\mathbf{y}}_{i}^{*}\right)$, EEV corresponds to: $E E V=\sum_{i \in I} \sum_{w \in \Omega_{i}} p_{i}^{(w)} \mathcal{Q}\left(\overline{\mathbf{x}}^{*}, \overline{\mathbf{y}}_{i}^{*}, \xi_{i}(w)\right)$. A large VSS means that uncertainty is important for the quality of the resulting optimal solution. On the contrary, a small VSS means that, a deterministic approach based on the expected values of the random variables might be sufficiently good to take a decision.

\subsection{Algorithmic Refinements}

The performance of the multi-cut integer L-shaped algorithm is improved by including some algorithmic refinements. We first discuss how to strength formulation (19)-(31) by adding valid inequalities and by lifting some of the existing constraints. Then, we study a special case to the problem and derive some properties that will help to simplify the complexity of the algorithm.

Lifting constraints in the first-stage problem: Constraints (21) can be lifted if the maximum number of o-d pairs to visit for a given courier $c \in C$, in a given time period $i \in I$, is greater than or equal to two $\left(\alpha_{i}^{c} \geq 2\right)$. The lifting procedure returns new values for the maximum number of o-d pairs to cover at each time period $i$ by each courier $c$, as well as new coefficients accompanying variables $y_{i p}^{c}$ in constraints (21). These values are denoted by $\alpha_{i}^{c *}$ and $\chi_{i p}^{c}$, respectively. This procedure is presented in Appendix A. With these new values, $\alpha_{i}^{c *}$ and $\chi_{i p}^{c}$, constraints (21) are replaced by:

$$
\begin{array}{r}
\sum_{p \in P} \chi_{i p}^{c} y_{i p}^{c} \leq \alpha_{i}^{c *} \sum_{s \in S^{c}} \delta_{i s}^{c} x_{s}^{c}, \forall c \in C, i \in I, \\
\sum_{p \in P} y_{i p}^{c} \geq \sum_{s \in S^{c}} \delta_{i s}^{c} x_{s}^{c}, \forall c \in C, i \in I .
\end{array}
$$

Adding valid cuts to the first-stage problem: Additional valid cuts can be added to the firststage problem (19)-(31) when $\alpha_{i}^{c *}=2$ and $\chi_{i p}^{c}=1$, to strengthen its lower bound. These cuts are defined as follows:

$$
y_{i p}^{c} \leq \sum_{s \in S^{c}} \delta_{i s}^{c} x_{s}^{c}, \forall c \in C, i \in I, p \in P \mid \alpha_{i}^{c *}=2, \chi_{i p}^{c}=1
$$

Special case: A special case of model RSLA is defined when the maximum number of o-d pairs to cover by the couriers at each time period is equal to one $\left(\alpha_{i}^{c *}=1\right)$. With this in mind, we define $R S L A^{\prime}$ as a relaxation of problem (19)-(31), in which integrality constraints (28) on first-stage variables $x_{s}^{c}$ are relaxed for all couriers with $\alpha_{i}^{c *}=1, \forall i \in I$. We have the following results:

Property 1. It always exists an optimal solution of problem $R S L A^{\prime}$ such that all $x_{s}^{c}$ variables take integer values.

Proof: For all courier $c \in C$ and time period $i \in I$ such that $\alpha_{i}^{c *}=1$ and $\chi_{i p}^{c}=1$, constraints 32 and 33 become: 


$$
\sum_{p \in P} y_{i p}^{c}=\sum_{s \in S^{c}} \delta_{i s}^{c} x_{s}^{c}, \forall c \in C, i \in I
$$

Note that $\sum_{p \in P} y_{i p}^{c} \in\{0,1\}$ for all $i \in I$, because $y_{i p}^{c} \in\{0,1\}, c \in C, i \in I, p \in P$, and $\alpha_{i}^{c *}=1, \forall i \in I$. Moreover, since $\sum_{s \in S^{c}} x_{s}^{c} \leq 1, \forall c \in C$, we have $\sum_{s \in S^{c}} \delta_{i s}^{c} x_{s}^{c} \leq 1, \forall c \in$ $C, i \in I$.

Since shifts $S^{c}, c \in C$ are different, i.e. shifts differ in at least one working period, an integer solution for $y_{i p}^{c}, c \in C, i \in I, p \in P$, implies that the solution for all $x_{s}^{c}, c \in C, s \in S^{c}$ variables will also be integer.

Property 2. If $\alpha_{i}^{c *}=1, \forall c \in C$ for a given $i \in I$, second-stage problems (13)-(18) can also be decomposed by o-d pairs:

When $\alpha_{i}^{c *}=1$ for each $c \in C$ at a given $i \in I$, constraints 15 become redundant since $\sum_{p \in P} y_{i p}^{c} \leq 1$ and $\mu_{i p}^{c} \bar{y}_{i p}^{c} \leq \beta_{i}^{c}$. Consequently, the resulting second-stage problem (13)(18) for time period $i \in I$ and scenario $w \in \Omega_{i}$ is decomposed into $|P|$ independent sorting problems, denoted by $\mathcal{Q}\left(\mathbf{x}, \mathbf{y}_{i p}, \xi_{i}(w)\right)$. The reformulation of the two-stage stochastic problem $R S L A$ under this condition, is presented in Appendix B.

\section{Operational Planning}

In this section, we describe the incorporation of the tactical master plan solutions into the operational planning for the real-time allocation of client requests to the couriers. We first make some assumptions and introduce some notation. Then, we propose a mathematical model and describe the solution approach adopted. We remark that the main objective of this work is to propose a robust tactical plan for attended home delivery problems. Hence, the goal of the approach presented in this Section is only to provide a tool to evaluate the robustness of the tactical plan, i.e. how well this plan reacts to new order requests arriving in real-time. The proposed method to tackle the operational planning is one possible approach, developing and evaluating different methods for real-time allocation of packages to the couriers is out of the scope of this work.

In defining a model and a solution method for the dynamic allocation of client requests to the couriers, we make the following assumptions. First, client requests can be consolidated to reduce transportation distances, time, and costs. A pair of requests is consolidated if the coordinates for the origins, for the destinations or for both, the origins and destinations, are the same for the two requests. Second, because of the small sizes and low weight of client requests (packages), capacity constraints on transportation modes only depend on the number of packages. Last, client requests are never rejected, as external couriers have an infinitive capacity.

For a given instant of time $t$ (contained into a tactical time interval $i \in I$ ), let $O_{t}$ be the set of client requests waiting to be delivered. Set $O_{t}$ is divided into $O_{t}^{N}$ and $O_{t}^{A}$. Set $O_{t}^{N}$ corresponds to new requests, i.e. requests that have not been assigned yet. Set $O_{t}^{A}$ corresponds to already assigned requests, i.e. requests that have been assigned to a courier $c$ in a previous instant of time $t^{\prime}<t$, but have not been delivered yet. Requests in $O_{t}^{A}$ are assigned to exactly one courier $c$, and once they are assigned, they cannot be delivered by another courier $c^{\prime}$. Each request, $o \in O_{t}$, has an associated delivery time window in minutes $\left[t w_{o}^{l}, t w_{o}^{u}\right]$ and an origin destination pair (o-d pair) with their corresponding coordinates (e.g., latitude coordinates and longitude coordinates). Let $C_{t}$ be 
the set of available couriers at instant $t$, and let $O_{t}^{c}$ be the set of client requests available at instant $t$ and that courier $c$ can deliver. Set $O_{t}^{c}$ includes new client requests, $O_{t}^{N c} \subseteq O_{t}^{N}$, and already assigned requests, $O_{t}^{A c} \subseteq O_{t}^{A}$. Let $J_{t}^{c}$ define a valid set of delivery patterns for each courier $c \in C_{t}$ at instant $t$. A delivery pattern $J_{t}^{c}$ is defined as a sequence of o-d pairs coordinates to be visited by courier $c$, starting at $t$ and finishing $t+l w$, where $l w$ is defined as the length of the time window, in minutes, for the operational planning. Note that each delivery pattern $J_{t}^{c}$ for every courier $c$ must include all already assigned packages $O_{t}^{A c}$, i.e. once an order $o \in O_{t}$ is assigned to a courier $c \in c_{t}$, o has to be delivered by courier $c$.

The tactical planning solution is incorporated into the operational planning in two ways. First, the set of available couriers at instant $t, C_{t}$, is constructed according to the optimal shift schedule

allocation $\mathbf{x}^{c *}$ from model $(19)-(31)$. Second, set $O_{t}^{c}$ is created by including the already assigned requests to courier $c$ at instant $t\left(O_{t}^{A c}\right)$, as well as a subset of the new order requests $\left(O_{t}^{N}\right)$. This subset, denoted by $O_{t}^{N c}$, is created based on the location of each order $o \in O_{t}^{N}$. Specifically, a new client request $o$ arriving at $t$ is added to $O_{t}^{N c}$ if the coordinates for the origin and the destination of $o$, are included into a "relaxation" of the optimal o-d pair allocation $\mathbf{y}^{c *}$, at any time period $i$ contained in the time window $\left[t w_{o}^{l}, t w_{o}^{u}\right]$. The "relaxation" of the optimal o-d pair allocation contains the set of o-d pairs $p$ allocated to courier $c$ at time period $i$, as well as the set of neighbors $N_{p}$ for each $p$ (an o-d pair $p^{\prime}$ is a neighbor of $p$, if the geographic zone associated with the destination of $p$ is adjacent to the geographic zone associated with the origin of $p^{\prime}$ ). The operational planning problem, for a specified time window $[t, t+t w]$, is formulated as a set-partitioning-based model, denoted as $L A_{\mathcal{J}_{t}}$. Its notation and formulation is as follows.

\subsection{Mathematical Model}

\section{Parameters:}

\begin{tabular}{ll}
\hline$\delta_{o j}^{c}:$ & $\begin{array}{l}\text { Parameter that takes value } 1 \text { if delivery pattern } j \text { for courier } c \text { includes request } o \text {, and } \\
\text { assumes value } 0 \text { otherwise; }\end{array}$ \\
$c_{t j}^{c}:$ & Cost associated to the assignment of delivery pattern $j$, to courier $c$, at instant $t ;$ \\
$c_{t o}:$ & Cost associated to the assignment of request $o$ to an external courier at instant $t$. \\
\hline
\end{tabular}

Table 2 - Notation for the operational planning model.

The decision variables for the operational planning are represented by $x_{t j}^{c}$ and $z_{t o}$. Variable $x_{t j}^{c}$ takes value 1 if delivery pattern $j$ is assigned to courier $c$ at instant $t$, and assumes value 0 otherwise. Variable $z_{\text {to }}$ takes value 1 if request $o \in O_{t}$ is delivered by an external courier at moment $t$, and assumes value 0 otherwise. The formulation of the operational planning problem is as follows: 


$$
\begin{aligned}
\left(L A_{\mathcal{J}_{t}}\right) \min & \sum_{c \in C_{t}} \sum_{j \in J_{t}^{c}} c_{t j}^{c} x_{t j}^{c}+\sum_{o \in O_{t}} c_{t o} z_{t o} \\
& \sum_{c \in C_{t}} \sum_{j \in J_{t}^{c}} \delta_{o j}^{c} x_{t j}^{c}+z_{t o}=1, \forall o \in O_{t}, \\
& \sum_{j \in J_{t}^{c}} x_{t j}^{c} \leq 1, \forall c \in C_{t}, \\
& z_{t o}=0, \forall o \in O_{t}^{A}, \\
& z_{t o} \geq 0, \forall o \in O_{t}^{N}, \\
& x_{t j}^{c} \in\{0,1\}, \forall c \in C_{t}, j \in J_{t}^{c} .
\end{aligned}
$$

The objective of $L A_{\mathcal{J}_{t}},(36)$, is to minimize the client request assignment cost for the dedicated couriers and external couriers. Constraints (37) guarantee that each request $o \in O_{t}$, is served by either a dedicated courier or by an external courier. Constraints (38) guarantee that every courier is assigned to at most one delivery pattern. Constraints (39) set $z_{\text {to }}$ to zero for the already assigned requests. Next section presents a description of the method used to generate the delivery patterns each time a new order request arrives to the system.

\subsection{Delivery Pattern Generation}

Delivery patterns $j \in J_{t}^{c}, \forall c \in C_{t}$ are updated each time a new event occurs in the system (e.g., the arrival of a new client request). These delivery patterns correspond to elementary resourceconstrained shortest paths with time windows in a directed graph $G_{t}^{c}=\left(\mathcal{N}_{t}^{c}, \mathcal{A}_{t}^{c}\right)$, where the set of nodes corresponds to $\mathcal{N}_{t}^{c}=\left\{n_{o} \mid o \in O_{t}^{c}\right\} \cup\left\{n_{s}, n_{f}\right\}, n_{o}$ represents the node assigned to client request $o$ and $n_{s}, n_{f}$ are the source and sink nodes, respectively. The set of arcs $\mathcal{A}_{t}^{c}$ is divided into three types: $\mathcal{A}_{1}$, arcs connecting the source node $n_{s}$ with request node $n_{o} ; \mathcal{A}_{2}$, arcs connecting two request nodes $n_{o}, n_{o^{\prime}} ; \mathcal{A}_{3}$, arcs connecting request node $n_{o}$ with the sink node $n_{f}$. Each node $n$ in $G_{t}^{c}=\left(\mathcal{N}_{t}^{c}, \mathcal{A}_{t}^{c}\right)$ has, besides a list of immediate successors $\mathcal{N}_{t}^{c}\left(n_{k}\right)=\left\{n_{o} \in \mathcal{N}_{t}^{c} \mid(k, o) \in \mathcal{A}_{t}^{c}\right\}$, a set of properties: its delivery time window, $\left[t w_{o}^{l}, t w_{o}^{u}\right]$, and a number of mandatory nodes that has to be visited before arriving to $n$. This last parameter is greater than zero only for the sink node, and its value represents the number of orders that have been already assigned to courier $c$. Similarly, each arc in $G_{t}^{c}=\left(\mathcal{N}_{t}^{c}, \mathcal{A}_{t}^{c}\right)$ has an associated set of properties: traversal cost, traversal time, and a Boolean parameter that takes value 1 if the head of the arc visits a mandatory node and assumes value 0 otherwise. A node $n \in \mathcal{N}_{t}^{c}$ is mandatory if the node corresponds to a request $o$ that has already been assigned to courier $c$.

To solve the elementary shortest path problem with resource constraints, we used a priorityqueue-based label-setting algorithm. At each iteration, this algorithm picks a label $l$ from a priority queue (the set of unprocessed labels) and checks it for dominance against the current set of labels at the node $n$ where $l$ resides. If $l$ is dominated by some other label residing at node $n, l$ is removed from the priority queue. If $l$ is non-dominated, it is extended along all immediate successors of $n$. Each resulting new label is checked for feasibility, and if it is feasible, it is added to the set of unprocessed labels, as well as to the set of labels residing at the respective successor node of $n$. If a new label is not feasible, it is discarded. The feasibility step verifies that all the constraints for the generation of patterns are met. These constraints are associated with the time windows for the delivery of each request, the minimum and maximum number of orders to include in each delivery pattern, the mandatory number of packages, and the availabilities of couriers. The algorithm stops 
when there are no more unprocessed labels or when a maximum number of delivery patterns have been found.

\section{Computational Experiments}

In this section, we test the proposed approach on real-world based instances for the integrated shift scheduling and load assignment optimization problem. First, we analyze some historical data and present information related to the operations in Colisweb. Second, we describe the procedure adopted for the generation of the instances. Third, we report and analyze the computational results.

The computational experiments were performed on a Linux operating system, 16 GB of RAM and 1 processor Intel Xeon X5675 running at 3.07GHz. The algorithms to solve the tactical and operational planning problems were implemented in C++. Problems (19)-(31) and (36)-(41) were solved with CPLEX version 12.6.0.1. Optimality cuts 23 were added to the problem as lazy constraints via CPLEX callbacks. We set a 1-hour and 2-hours time limit to solve each instance of tactical problem (19)-(31) when $\alpha_{i}^{c}$ is set to one and when $\alpha_{i}^{c}$ is set to two, respectively. A relative gap tolerance of 0.01 was set as a stopping criterion for solving the MILPs with CPLEX. The value of tolerance $\epsilon_{1}$ (tolerance to stop the McDaniel and Devine (1977) strategy) was set to 0.05 .

\subsection{Operations and Client Orders Characteristics}

As mentioned above, this study is motivated by operations at Colisweb, a company that offers lastmile delivery services in almost all major cities in France. The test instances are generated based on 6-months historical data from operations in the metropolitan region of Paris. This region has a service area of $762.4 \mathrm{~km}^{2}$, with $|A|=67$ geographic zones corresponding to postal codes, and $|P|=1,705$ potential number of o-d pairs, classified as center (inside of Paris) or suburb (outside of Paris).

Colisweb operates in Paris from Monday to Saturday from 7:00 to 21:00. Hence the tactical planning horizon is divided into 14 time periods of one-hour length. Two types of couriers are considered: dedicated and external. Dedicated couriers utilize three types of vehicles to serve client requests: bicycles, motorcycles, and small cars. External couriers have an unlimited delivery capacity and are always available. Delivery costs for external couriers depend on the type of o-d pairs served, as well as on the number of packages delivered. Thus, the cost of delivering one package in center region is 35 , while the cost of delivering one package in suburb region is 45 . Each dedicated courier has different availabilities regarding the time he can start and end its working shift. Hence, shifts for a given courier $c$ can start at any time period of the courier's availability, allowing enough time to complete their duration. We considered 4 types of shifts: 4-hour, 5-hour, 6-hour, and 8-hour shifts with no breaks. The values for the delivery capacity $\beta_{i p}^{c}$, the total capacity $\beta_{i}^{c}$, the fixed $\operatorname{cost} f_{i}^{c}$, the variable cost $l_{i p}^{c}$, and the maximum distance $\tau_{i}^{c}$ for courier $c$ depend on the type of vehicle used. These values are presented in Table 3. In addition to these values we introduce three new operational parameters, $\gamma_{o}, \gamma_{d}$, and $\zeta$, representing the service time required to pick-up a request, the service time required to deliver a request, and the order consolidation factor, respectively. These values are set to 10 minutes, 10 minutes, and to 0.5 , respectively. For confidentiality reasons the collected data, in particular the cost information, has been modified without loss of scientific relevance for the problem. 


\begin{tabular}{lccc}
\hline Parameter & Bicycle & Motorcycle & Car \\
\hline$\beta_{i p}^{c}$ & 3 & 3 & 4 \\
$\beta_{i}^{c}$ & 3 & 3 & 4 \\
$f_{i}^{c}$ & 0 & 10 & 15 \\
$l_{i p}^{c}$ & 15 & 5 & 5 \\
$\tau_{i}^{c}$ & $15 \mathrm{~km} / \mathrm{hr}$ & $20 \mathrm{~km} / \mathrm{hr}$ & $30 \mathrm{~km} / \mathrm{hr}$ \\
\hline
\end{tabular}

Table 3 - Capacities, costs and maximum distance values for each type of vehicle.

\subsection{Instances and Scenario Generation}

We generated two types of client orders: same-day instant delivery (S-D) and next-day delivery (N-D). These types of orders have service time windows of 2 hours. All N-D orders are known at the beginning of each day. On the contrary, S-D orders are not known at the beginning of the day, as they arrive dynamically with time. The percentage of S-D and N-D orders ready to be assigned to a courier varies significantly depending on the period of the day, as it can be observed in Table 4. For instance, S-D requests are more likely to appear 3 hours after the operations start and its percentage tends to increase with the periods of the day. On the contrary, the percentage of N-D deliveries shows a decrease and then a stabilization with the periods of the day.

\begin{tabular}{|c|c|c|c|c|c|c|c|c|c|c|c|c|c|c|}
\hline \multirow[b]{2}{*}{ Order type } & \multicolumn{14}{|c|}{ Time period } \\
\hline & $7: 00$ & 8:00 & 9:00 & 10:00 & $11: 00$ & 12:00 & $13: 00$ & $14: 00$ & $15: 00$ & 16:00 & $17: 00$ & $18: 00$ & 19:00 & 20:00 \\
\hline S-D & 0 & 0 & 2.5 & 5 & 26.5 & 52.8 & 63.2 & 48 & 66 & 60 & 65 & 55.4 & 82 & 100 \\
\hline N-D & 100 & 100 & 97.5 & 95 & 73.5 & 47.2 & 36.8 & 52 & 34 & 40 & 35 & 44.6 & 18 & 0 \\
\hline
\end{tabular}

Table 4 - Order percentage depending on the time period and order type.

A large set of real-world based instances were randomly generated to test our method. The deterministic demand patterns for the tactical planning were created such that they follow two shapes $(F)$ : unimodal $(U)$ and bimodal $(B)$. These shapes are representative of sectors that have a single peak or two peaks of demand during each day, respectively. We also considered five levels for the total number of orders to deliver per day $(V)$. For each combination of $(F, V)$, we generated 10 different demand profiles. Therefore, the resulting number of instances for the deterministic demand patterns is 100 (50 deterministic demand patterns for the unimodal shape and 50 deterministic demand patterns for the bimodal shape). From these patterns, we compute the total number of orders $d_{i}$ to deliver at each time period $i \in I$. This total number of orders $d_{i}$ is divided into two parts: a part corresponding to the deterministic demand (N-D type of orders) and a part corresponding to the mean of the stochastic demand (S-D type of orders). This division is done according to the values from Table 4 . Figures 1 and 2 present two examples corresponding to a unimodal and a bimodal distribution of order requests. These figures also present the division of the total demand into two types: N-D (deterministic orders) and S-D (stochastic orders).

Let $\bar{\xi}_{i}$ denote the mean for the total number of S-D client requests at each time period $i \in I$. Let $n d_{i}$ denote the number of N-D requests at time period $i$. For each postal code $a \in A$, let $p_{a o}$ and $p_{a d}$ denote the probabilities of being selected as an origin $o$ or a destination $d$, respectively. We assume that S-D delivery requests arrive to the system according to a Poisson distribution with mean $\bar{\xi}_{i}$, and that N-D deliveries are known in advance; hence the total number of client requests at each time period for N-D deliveries is the same for all the scenarios. Attended home delivery companies 


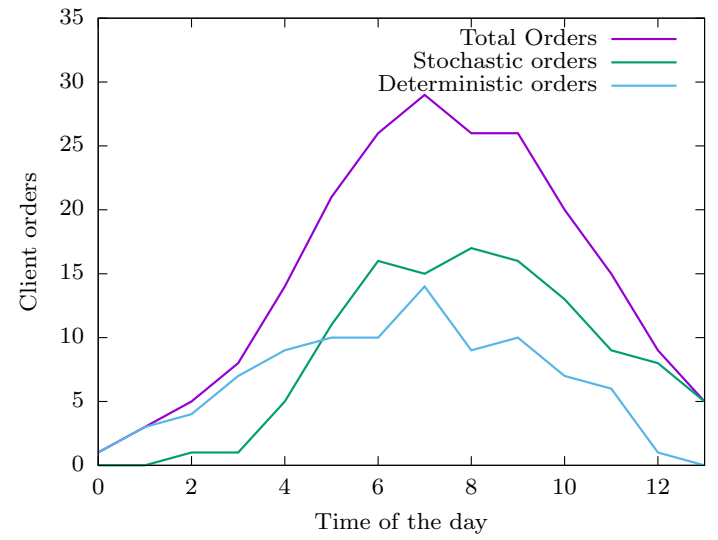

Figure 1 - Unimodal distribution for client orders.

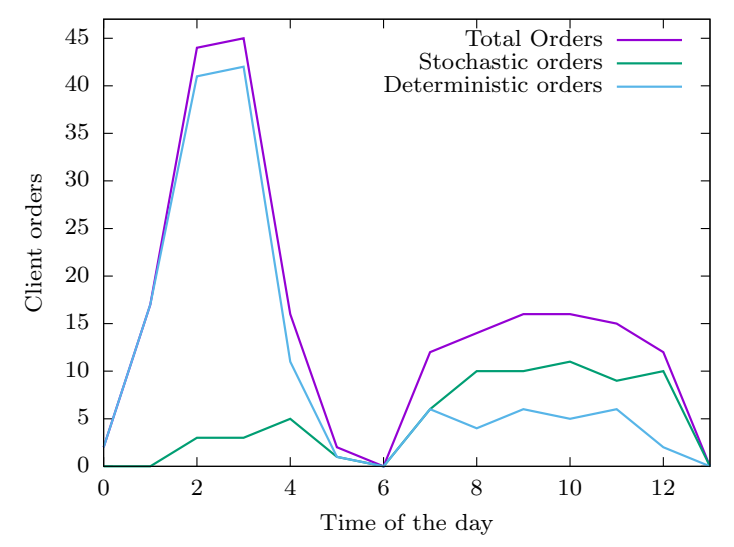

Figure 2 - Bimodal distribution for client orders.

normally experiment strong time-varying arrival rates. Thus, a Poisson process is a natural way to model these arrival processes. The description of the steps followed to generate the tactical and operational instances follows.

\subsubsection{Generation of tactical instances.}

The generation of the different stochastic scenarios for each tactical instance is done in two steps. In the first step, we generate all possible demand realizations for each time period. In the second step, we allocate the client requests (deterministic and stochastic) to o-d pairs.

1. Scenario generation for the total demand: In this step, we generate all possible demand realizations $\xi_{i}^{(w)}$ for the S-D requests at each time period $i \in I$. These scenarios are related to a positive or to a negative perturbation of demand $\kappa_{i}^{(w)}=\xi_{i}^{(w)}-\bar{\xi}_{i}$, i.e. the perturbation is positive if $\xi_{i}^{(w)} \geq \bar{\xi}_{i}$ and negative if $\xi_{i}^{(w)}<\bar{\xi}_{i}$. In this step, we also compute the probability $p_{i}^{(w)}$ for each scenario $w \in \Omega_{i}$. The value of $p_{i}^{(w)}$ is given by the probability mass function for a Poisson distribution.

2. Spacial distribution of client orders: This step is divided into two phases. In the first phase, we allocate each deterministic client request ( $\mathrm{N}-\mathrm{D}$ requests and requests corresponding to the mean for S-D requests) to the set of o-d pairs. We recall that the tactical instances do not consider the specific coordinates for the origin and destination of client requests, instead we aggregate them depending on the geographic zone, corresponding in our case to postal codes. This allocation is done according to the probabilities $p_{a o}$ and $p_{a d}$ and to one criterion denoted as "order density" $(\rho)$. This criterion determines the number of orders allocated to each o-d pair at each time period. If $\rho=U\{1,1\}$, the demand is considered as "disperse" (Dis.). On the contrary, if $\rho=U\{1,3\}$ the demand is considered as "dense" (Den.). Hence, we first randomly generate an origin $o$ and a destination $d$ that determines o-d pair $p$, we then generate the value for the density of the demand. If the instance considers a disperse demand, we assign one order to o-d pair $p$. Otherwise, we generate a random variable $\rho$ with a discrete uniform distribution between one and three and we assign these $\rho$ packages to o-d pair $p$. This process is repeated until the total demand $d_{i}=n d_{i}+\bar{\xi}_{i}$ at each time period $i \in I$ is assigned to the o-d pairs. In the second phase, we allocate the stochastic part of the client requests to the set 
of o-d pairs. We define two options for the spatial distribution of these stochastic requests: a random $(R)$ spatial distribution and a non-random (NR) spatial distribution. If $R$ is chosen and the stochastic perturbation of the demand is positive $\left(\kappa_{i}^{(w)}>0\right)$, we randomly generate $\kappa_{i}^{(w)}$ new o-d pairs with one client order. On the contrary, if NR is selected, we randomly choose $\kappa_{i}^{(w)}$ o-d pairs from the ones generated in the first phase, and add one order to each one of them. If the stochastic perturbation of the orders is negative $\left(\kappa_{i}^{(w)}<0\right)$, we randomly choose $\kappa_{i}^{(w)}$ o-d pairs from the ones generated in the first phase, and remove one order from each one of them.

Ten different demands were tested for six combinations (Combin.) of demand shape $(U, B)$, total number of client requests $(V)$, demand density (Dis., Den.), and spatial distribution $(R, N R)$. Table 5 shows the size of these instances. The average values for the minimum and maximum number of scenarios at each time period are presented in Columns 2 and 3. These values are labeled as Min.Scen. and Max.Scen., respectively. The average number of o-d pairs $|P|$ with at least one client request over all time periods and scenarios is presented in Column 5. Column 6, labeled as Nb.Packages, presents the average number of requests during the planning horizon. The average value for the density (Density) is presented in Column 6. Density is computed as Density $=$ Nb.Packages $/|P|$. The average number of couriers $(|C|)$ and the average value for the total number of working shifts (Nb.Shifts) are presented in the last two columns.

\subsubsection{Generation of operational instances.}

Each operational instance is generated as a random realization of its corresponding tactical instance. In particular, each one of these operational instances is composed by two types of client requests: N-D orders and S-D orders. As in the tactical planning, N-D orders are known in advance, and S-D orders arrive to the system according to a Poisson process with time-dependent rates $\bar{\xi}_{i}, i \in I$. Note that each client order $o \in O_{t}^{N}$ is characterized by a type of order (N-D or S-D), by an arrival time $t_{o}$, by a length of the time window $l w_{o}$, and by an origin destination pair (o-d pair) with their corresponding latitude and longitude coordinates, denoted as $\left(\left(\operatorname{lat}_{o}^{o}, \operatorname{lon}_{o}^{o}\right),\left(\operatorname{lat}_{o}^{d}, \operatorname{lon}_{o}^{d}\right)\right)$, respectively. Additionally, observe that each one of these client orders $o \in O_{t}^{N}$ have a tactical equivalent, with a corresponding arrival time period denoted by $i$. The procedure to generate the values for $t_{o}$, as well as the values for $\left(\left(\operatorname{lat}_{o}^{o}, \operatorname{lon}_{o}^{o}\right),\left(\operatorname{lat}_{o}^{d}, \operatorname{lon}_{o}^{d}\right)\right)$ for all $o \in O_{t}$ is as follows.

1. Arrival time generation: We created the arrival time $t_{o}$, for each order $o$, by generating Exponential variables with a mean equals to the inverse of the Poisson order arrival rate (converted to minutes). Specifically, the arrival time for each N-D type of orders in $i \in I$ is generated with an Exponential variable with mean $60 / n d_{i}$. Since each operational instance is a random realization of its tactical counterpart we first generate, for each $i \in I$, a Poisson-distributed random variable with mean $\bar{\xi}_{i}$. This variable, denoted as $s d_{i}$, corresponds to the number of same-day client requests at time period $i$. We then generate the arrival time for each order in $s d_{i}$, with an Exponential variable with mean $60 / s d_{i}$.

2. Origin-destination coordinates generation: We created the latitude and longitude coordinates for the origin and for the destination of each order by generating points uniformly, randomly, and independently distributed within a circle of radius $r$ around a location $(x 0, y 0)$. The value of $r$ corresponds to the radius of the geographic zone associated to the origin or to the destination of order $o$. Coordinates $(x 0, y 0)$ are given by the tactical instances, and their values correspond to the latitude and longitude coordinates of the centroid of the geographic zone associated to the origin or to the destination of order $o$. 


\begin{tabular}{|c|c|c|c|c|c|c|c|c|}
\hline Combin. & Version & Min.Scen. & Max.Scen. & $|P|$ & Nb.Packages & Density & $|C|$ & Nb.Shifts \\
\hline \multirow{5}{*}{ U/Den./R } & 0 & 3 & 16 & 197 & 50 & 0.25 & 9 & 333 \\
\hline & 1 & 3 & 24 & 323 & 89 & 0.28 & 16 & 588 \\
\hline & 2 & 3 & 31 & 411 & 128 & 0.31 & 23 & 836 \\
\hline & 3 & 3 & 34 & 474 & 168 & 0.35 & 30 & 1,091 \\
\hline & 4 & 3 & 38 & 538 & 206 & 0.38 & 36 & 1,339 \\
\hline \multirow{5}{*}{ B/Den./R } & 0 & 3 & 12 & 143 & 74 & 0.52 & 23 & 851 \\
\hline & 1 & 3 & 18 & 225 & 110 & 0.49 & 32 & 1,195 \\
\hline & 2 & 3 & 24 & 284 & 143 & 0.5 & 42 & 1,539 \\
\hline & 3 & 3 & 27 & 342 & 177 & 0.52 & 51 & 1,902 \\
\hline & 4 & 3 & 30 & 373 & 212 & 0.57 & 62 & 2,275 \\
\hline \multirow{5}{*}{ U/Dis./NR } & 0 & 3 & 15 & 79 & 52 & 0.66 & 9 & 337 \\
\hline & 1 & 3 & 24 & 146 & 92 & 0.63 & 16 & 581 \\
\hline & 2 & 3 & 30 & 181 & 131 & 0.72 & 23 & 832 \\
\hline & 3 & 3 & 34 & 193 & 171 & 0.89 & 29 & 1,073 \\
\hline & 4 & 3 & 37 & 201 & 208 & 1.03 & 36 & 1,328 \\
\hline \multirow{5}{*}{ U/Den./NR } & 0 & 3 & 16 & 54 & 56 & 1.04 & 9 & 333 \\
\hline & 1 & 3 & 25 & 98 & 98 & 1.0 & 16 & 588 \\
\hline & 2 & 3 & 30 & 123 & 135 & 1.1 & 22 & 829 \\
\hline & 3 & 3 & 34 & 134 & 176 & 1.31 & 30 & 1,095 \\
\hline & 4 & 3 & 38 & 137 & 215 & 1.57 & 37 & 1,350 \\
\hline \multirow{5}{*}{ B/Dis./NR } & 0 & 3 & 12 & 81 & 77 & 0.95 & 23 & 851 \\
\hline & 1 & 3 & 18 & 124 & 113 & 0.91 & 33 & 1,202 \\
\hline & 2 & 3 & 24 & 159 & 146 & 0.92 & 42 & 1,535 \\
\hline & 3 & 3 & 27 & 181 & 180 & 0.99 & 52 & 1,905 \\
\hline & 4 & 4 & 29 & 197 & 214 & 1.09 & 61 & 2,261 \\
\hline \multirow{5}{*}{ B/Den./NR } & 0 & 3 & 12 & 55 & 79 & 1.44 & 23 & 847 \\
\hline & 1 & 3 & 18 & 83 & 117 & 1.41 & 33 & 1,206 \\
\hline & 2 & 3 & 23 & 101 & 150 & 1.49 & 42 & 1,535 \\
\hline & 3 & 3 & 27 & 121 & 184 & 1.52 & 52 & 1,917 \\
\hline & 4 & 4 & 30 & 132 & 219 & 1.66 & 61 & 2,253 \\
\hline
\end{tabular}

Table 5 - Size of the stochastic tactical instances.

\subsection{Computational Results}

Table 6 presents the computational results on stochastic instances for the integrated shift scheduling and load assignment tactical problem for $\alpha_{i}^{c}=1$ and for $\alpha_{i}^{c}=2, \forall c \in C, i \in I$. These results include the average CPU time in seconds to solve the problem (T.time(s.)), the average number of optimality cuts added to the problem $(\mathrm{Nb} . \mathrm{Cuts})$, the number of instances that converged to a near-optimal solution (Conv.), i.e. the algorithm stopped when no more optimality cuts were added to the first-stage model. The average gap between the best upper bound and the best lower bound (Gap). This gap is computed as: Gap $=100 \times\left(\bar{z}-\left(\mathbf{f}^{T} \mathbf{x}_{k}^{*}+\underline{Q}^{k}\right)\right) / \bar{z}$. The percentage of the total cost spent in external couriers $\left(\right.$ Ext.C), computed as: Ext. $C=100 \times\left(\sum_{i \in I} \sum_{w \in \Omega_{i}} \sum_{p \in P} c_{i p} e_{i p}^{(w) *}\right) / \bar{z}$. Where $e_{i p}^{(w) *}$ represents the optimal solution for the number of external couriers used at time period $i$, in o-d pair $p$, under scenario $w$. Table 6 also presents the average impact in the total cost when $\alpha_{i}^{c}$ is increased from one to two. This value is computed as: $1 v s .2=100 \times\left(\bar{z}_{1}-\bar{z}_{2}\right) / \bar{z}_{1}$, where $\bar{z}_{1}$ and $\bar{z}_{2}$ correspond to the final upper bound value when the problem is solved with $\alpha_{i}^{c}=1$ and $\alpha_{i}^{c}=2$, $\forall c \in C, i \in I$, respectively. A negative value in Column $1 v s .2$ means that a feasible solution was found for the problem when $\alpha_{i}^{c}=2$, but this value is higher than the cost found for the problem when $\alpha_{i}^{c}=1$. The character "-" in columns Gap, Ext.C, and 1vs.2 means that the method was not 
able to find an integer solution for any of the 10 instances tested.

\begin{tabular}{|c|c|c|c|c|c|c|c|c|c|c|c|c|}
\hline \multirow[b]{2}{*}{ Combin. } & \multirow[b]{2}{*}{ Version } & \multicolumn{5}{|c|}{$\alpha=1$} & \multicolumn{5}{|c|}{$\alpha=2$} & \multirow[b]{2}{*}{$1 v s .2$} \\
\hline & & T.time(s.) & Nb.Cuts & Conv. & Gap & Ext.C & T.time (s.) & Nb.Cuts & Conv. & Gap & Ext.C & \\
\hline \multirow{5}{*}{ U/Den./R } & 0 & 9.90 & 1,471 & 10 & $0.16 \%$ & $69.00 \%$ & 28.57 & 5,257 & 10 & $0.34 \%$ & $65.57 \%$ & $2.78 \%$ \\
\hline & 1 & 51.85 & 3,910 & 10 & $0.25 \%$ & $66.66 \%$ & 186.96 & 16,167 & 10 & $0.42 \%$ & $64.94 \%$ & $3.08 \%$ \\
\hline & 2 & 155.49 & 11,976 & 10 & $0.34 \%$ & $65.48 \%$ & 885.44 & 36,614 & 10 & $0.48 \%$ & $62.97 \%$ & $4.12 \%$ \\
\hline & 3 & 310.07 & 16,686 & 10 & $0.02 \%$ & $66.51 \%$ & $3,618.79$ & 59,606 & 7 & $0.90 \%$ & $64.24 \%$ & $4.03 \%$ \\
\hline & 4 & 527.19 & 23,668 & 10 & $0.02 \%$ & $65.10 \%$ & $7,133.13$ & 85,122 & 2 & $6.67 \%$ & $62.57 \%$ & $-2.79 \%$ \\
\hline \multirow{5}{*}{ B/Den./R } & 0 & 19.76 & 1,535 & 10 & $0.36 \%$ & $39.77 \%$ & 123.44 & 2,354 & 10 & $0.22 \%$ & $38.12 \%$ & $3.18 \%$ \\
\hline & 1 & 88.60 & 4,523 & 10 & $0.32 \%$ & $39.59 \%$ & 573.15 & 5,808 & 10 & $0.28 \%$ & $38.58 \%$ & $2.18 \%$ \\
\hline & 2 & 225.80 & 6,699 & 10 & $0.15 \%$ & $38.56 \%$ & $3,790.71$ & 15,885 & 10 & $0.23 \%$ & $37.02 \%$ & $3.43 \%$ \\
\hline & 3 & 543.87 & 10,729 & 10 & $0.21 \%$ & $39.59 \%$ & $5,659.21$ & 23,345 & 2 & $11.68 \%$ & $42.22 \%$ & $-13.50 \%$ \\
\hline & 4 & 989.04 & 20,914 & 10 & $0.31 \%$ & $40.30 \%$ & $6,862.59$ & 25,705 & 0 & - & - & - \\
\hline \multirow{5}{*}{ U/Dis./NR } & 0 & 5.16 & 2,357 & 10 & $0.05 \%$ & $1.11 \%$ & 14.45 & 2,454 & 10 & $0.26 \%$ & $1.17 \%$ & $4.28 \%$ \\
\hline & 1 & 44.42 & 6,745 & 10 & $0.00 \%$ & $0.48 \%$ & 128.15 & 7,215 & 10 & $0.15 \%$ & $0.49 \%$ & $4.74 \%$ \\
\hline & 2 & 149.84 & 11,276 & 10 & $0.09 \%$ & $0.38 \%$ & 437.86 & 12,244 & 10 & $0.08 \%$ & $0.19 \%$ & $3.97 \%$ \\
\hline & 3 & 325.21 & 18,889 & 10 & $0.12 \%$ & $0.34 \%$ & $1,047.65$ & 20,027 & 10 & $0.11 \%$ & $0.12 \%$ & $3.92 \%$ \\
\hline & 4 & 494.02 & 23,022 & 10 & $0.10 \%$ & $0.28 \%$ & $3,213.85$ & 39,276 & 10 & $0.21 \%$ & $0.24 \%$ & $4.54 \%$ \\
\hline \multirow{5}{*}{ U/Den./NR } & 0 & 2.28 & 1,265 & 10 & $0.20 \%$ & $2.18 \%$ & 16.09 & 2,799 & 10 & $0.65 \%$ & $2.12 \%$ & $1.58 \%$ \\
\hline & 1 & 16.72 & 3,187 & 10 & $0.45 \%$ & $1.62 \%$ & 530.62 & 10,458 & 10 & $0.77 \%$ & $1.06 \%$ & $2.35 \%$ \\
\hline & 2 & 65.41 & 5,229 & 10 & $0.26 \%$ & $1.23 \%$ & $1,776.50$ & 18,151 & 9 & $0.90 \%$ & $0.94 \%$ & $2.66 \%$ \\
\hline & 3 & 140.19 & 9,311 & 10 & $0.42 \%$ & $0.87 \%$ & $5,215.26$ & 24,141 & 5 & $6.47 \%$ & $6.66 \%$ & $-5.74 \%$ \\
\hline & 4 & 161.69 & 10,029 & 10 & $0.25 \%$ & $0.63 \%$ & $5,600.60$ & 25,177 & 3 & $5.55 \%$ & $5.62 \%$ & $-4.56 \%$ \\
\hline \multirow{5}{*}{ B/Dis./NR } & 0 & 14.14 & 2,287 & 10 & $0.01 \%$ & $17.23 \%$ & 51.18 & 2,367 & 10 & $0.34 \%$ & $18.74 \%$ & $3.48 \%$ \\
\hline & 1 & 51.41 & 5,448 & 10 & $0.15 \%$ & $13.25 \%$ & 574.56 & 5,521 & 9 & $0.53 \%$ & $13.94 \%$ & $3.34 \%$ \\
\hline & 2 & 123.71 & 8,007 & 10 & $0.02 \%$ & $11.31 \%$ & $1,079.79$ & 7,952 & 9 & $0.58 \%$ & $12.12 \%$ & $3.32 \%$ \\
\hline & 3 & 246.38 & 14,545 & 10 & $0.08 \%$ & $13.26 \%$ & $3,247.74$ & 17,839 & 10 & $0.08 \%$ & $14.29 \%$ & $3.13 \%$ \\
\hline & 4 & 689.19 & 24,335 & 10 & $0.18 \%$ & $11.87 \%$ & $4,789.92$ & 31,911 & 6 & $0.24 \%$ & $14.27 \%$ & $3.70 \%$ \\
\hline \multirow{5}{*}{ B/Den./NR } & 0 & 6.82 & 1,029 & 10 & $0.25 \%$ & $4.33 \%$ & 48.71 & 1,696 & 10 & $0.43 \%$ & $3.98 \%$ & $2.19 \%$ \\
\hline & 1 & 38.02 & 2,714 & 10 & $0.34 \%$ & $3.80 \%$ & 485.00 & 5,561 & 10 & $0.60 \%$ & $4.29 \%$ & $1.45 \%$ \\
\hline & 2 & 87.46 & 4,235 & 10 & $0.22 \%$ & $2.82 \%$ & $4,268.23$ & 12,031 & 9 & $1.07 \%$ & $2.67 \%$ & $2.79 \%$ \\
\hline & 3 & 180.61 & 5,438 & 10 & $0.27 \%$ & $3.05 \%$ & $6,235.23$ & 12,334 & 3 & $15.70 \%$ & $15.90 \%$ & $-20.13 \%$ \\
\hline & 4 & 248.44 & 8,862 & 10 & $0.19 \%$ & $2.75 \%$ & $7,200.41$ & 10,573 & 0 & $44.06 \%$ & $44.26 \%$ & $-80.37 \%$ \\
\hline
\end{tabular}

Table 6 - Computational effort on stochastic instances with $\alpha=1$ and $\alpha=2$.

From Table 6, we can conclude that our method was able to find solutions that converge for all the instances evaluated with $\alpha_{i}^{c}=1, c \in C, i \in I$. Our method was able to find good-quality solutions for the smaller instances when $\alpha_{i}^{c}$ is set to two $\forall i \in C, i \in I$, but for the larger instances, the method was not able to find solutions within the CPU time limit, due to the large number of o-d pairs and couriers. Regarding the values of Ext.C (percentage of the total cost spent in external couriers), we can conclude that they are very similar within all Combin. when $\alpha_{i}^{c}=1$ and when $\alpha_{i}^{c}=2$. However, these percentages are significantly different depending on the spatial distribution of new requests, i.e. random $(R)$ and not random $(N R)$. Specifically, the value of Ext.C is significantly larger for the instances generated with random spatial distribution of new requests, than for the instances generated with a non-random spatial distribution of new requests.

We observe that, the CPU time and the number of optimality cuts added to the problem increase with the numbers of couriers and o-d pairs. We also note that there is a significant difference in these values when $\alpha_{i}^{c}$ increases from one to two. The method takes in average less than 30 minutes to solve all the instances when $\alpha_{i}^{c}=1$. On the contrary, when $\alpha_{i}^{c}=2$, the method stops (the CPU time limit is reached) without being able to find any feasible integer solution, for some of the larger instances. Note that, despite this increase in the CPU time, allowing the couriers to cover more o-d pairs per time period causes a decrease in the total cost of the tactical planning, specially for the disperse (Dis.) instances where we observed an average decrease ranging from $3.13 \%$ up to $4.74 \%$. 


\subsection{Value of the Stochastic Solution}

Tables 7 and 8 present a comparison between the results and computational effort of the two-stage stochastic problem and the mean value problem when all $\alpha_{i}^{c}$ are set to one and to two, respectively. For each instance combination (Combin.) and number of packages (Version), we present the average CPU time in seconds to solve the two-stage stochastic programming problem (T.time(s.)) and the average CPU time required to solve the mean value problem (T.timeEV(s.)). We also report the average value of the stochastic solution associated with tree factors: shift allocation costs $\left(V S S_{F}\right)$, dedicated courier order allocation costs $\left(V S S_{D}\right)$, and external courier order allocation costs $\left(V S S_{E}\right)$. The average of the stochastic solution $\left(V S S_{T}\right)$ is presented in the last column. These values are computed as: $V S S_{c}=100 \times\left(E E V_{c}-R P_{c}\right) / E E V_{T}$, for all $c=\{T, F, D, E\}$, where $E E V_{T}, E E V_{F}$, $E E V_{D}$, and $E E V_{E}$ correspond to the expected value of the total cost, shift allocation cost, dedicated courier order allocation cost, and external courier order allocation cost when using the EV solution, respectively. In the same way, $R P_{T}, R P_{F}, R P_{D}$, and $R P_{E}$ correspond to the final value of the total cost, shift allocation cost, dedicated courier order allocation cost, and external courier order allocation cost when using the stochastic model, respectively. The character "-" in columns $V S S_{F}$, $V S S_{D}, V S S_{E}$, and $V S S_{T}$ means that the method was not able to find any integer solution for either one or the two problems (stochastic problem and mean value problem).

\begin{tabular}{|c|c|c|c|c|c|c|c|}
\hline Combin. & Version & T.time(s.) & T.timeEV(s.) & $V S S_{F}$ & $V S S_{D}$ & $V S S_{E}$ & $V S S_{T}$ \\
\hline \multirow{5}{*}{ U/Den./R } & 0 & 9.90 & 8.30 & $5.82 \%$ & $-0.81 \%$ & $5.16 \%$ & $10.17 \%$ \\
\hline & 1 & 51.85 & 49.29 & $3.21 \%$ & $-1.27 \%$ & $9.17 \%$ & $11.11 \%$ \\
\hline & 2 & 155.49 & 138.46 & $1.86 \%$ & $-0.72 \%$ & $9.75 \%$ & $10.89 \%$ \\
\hline & 3 & 310.07 & 288.36 & $2.13 \%$ & $-0.86 \%$ & $9.89 \%$ & $11.16 \%$ \\
\hline & 4 & 527.19 & 485.56 & $2.16 \%$ & $-1.30 \%$ & $11.41 \%$ & $12.27 \%$ \\
\hline \multirow{5}{*}{ B/Den./R } & 0 & 19.76 & 16.93 & $5.50 \%$ & $-4.57 \%$ & $11.62 \%$ & $12.55 \%$ \\
\hline & 1 & 88.60 & 49.89 & $0.07 \%$ & $-3.06 \%$ & $14.76 \%$ & $11.77 \%$ \\
\hline & 2 & 225.80 & 115.09 & $1.59 \%$ & $-3.38 \%$ & $14.56 \%$ & $12.78 \%$ \\
\hline & 3 & 543.87 & 241.30 & $1.77 \%$ & $-2.91 \%$ & $13.38 \%$ & $12.24 \%$ \\
\hline & 4 & 989.04 & 323.47 & $0.94 \%$ & $-2.56 \%$ & $14.80 \%$ & $13.17 \%$ \\
\hline \multirow{5}{*}{ U/Dis./NR } & 0 & 5.16 & 2.79 & $-0.18 \%$ & $3.12 \%$ & $0.52 \%$ & $3.46 \%$ \\
\hline & 1 & 44.42 & 20.49 & $0.00 \%$ & $2.33 \%$ & $0.61 \%$ & $2.94 \%$ \\
\hline & 2 & 149.84 & 66.22 & $0.46 \%$ & $2.65 \%$ & $0.21 \%$ & $3.32 \%$ \\
\hline & 3 & 325.21 & 124.95 & $0.16 \%$ & $2.20 \%$ & $0.51 \%$ & $2.88 \%$ \\
\hline & 4 & 494.02 & 164.42 & $0.04 \%$ & $2.15 \%$ & $0.65 \%$ & $2.85 \%$ \\
\hline \multirow{5}{*}{ U/Den./NR } & 0 & 2.28 & 1.32 & $-1.92 \%$ & $1.39 \%$ & $14.06 \%$ & $13.53 \%$ \\
\hline & 1 & 16.72 & 7.82 & $-2.73 \%$ & $0.10 \%$ & $18.47 \%$ & $15.83 \%$ \\
\hline & 2 & 65.41 & 22.83 & $-3.14 \%$ & $-0.22 \%$ & $19.52 \%$ & $16.16 \%$ \\
\hline & 3 & 140.19 & 39.65 & $-2.99 \%$ & $-0.07 \%$ & $19.28 \%$ & $16.22 \%$ \\
\hline & 4 & 161.69 & 55.84 & $-3.53 \%$ & $0.23 \%$ & $19.10 \%$ & $15.80 \%$ \\
\hline \multirow{5}{*}{ B/Dis./NR } & 0 & 14.14 & 8.00 & $11.35 \%$ & $1.77 \%$ & $-11.59 \%$ & $1.53 \%$ \\
\hline & 1 & 51.41 & 26.39 & $6.48 \%$ & $0.51 \%$ & $-5.78 \%$ & $1.21 \%$ \\
\hline & 2 & 123.71 & 58.76 & $2.35 \%$ & $0.07 \%$ & $-1.21 \%$ & $1.21 \%$ \\
\hline & 3 & 246.38 & 139.66 & $5.49 \%$ & $0.37 \%$ & $-4.48 \%$ & $1.38 \%$ \\
\hline & 4 & 689.19 & 173.58 & $2.56 \%$ & $-0.10 \%$ & $-0.87 \%$ & $1.59 \%$ \\
\hline \multirow{5}{*}{ B/Den./NR } & 0 & 6.82 & 3.67 & $1.80 \%$ & $-1.85 \%$ & $10.62 \%$ & $10.57 \%$ \\
\hline & 1 & 38.02 & 11.16 & $1.14 \%$ & $-2.13 \%$ & $12.29 \%$ & $11.30 \%$ \\
\hline & 2 & 87.46 & 25.29 & $3.26 \%$ & $-2.61 \%$ & $9.54 \%$ & $10.18 \%$ \\
\hline & 3 & 180.61 & 50.86 & $3.12 \%$ & $-3.10 \%$ & $10.41 \%$ & $10.42 \%$ \\
\hline & 4 & 248.44 & 73.11 & $1.43 \%$ & $-1.71 \%$ & $11.05 \%$ & $10.78 \%$ \\
\hline
\end{tabular}

Table 7 - Value of the stochastic solution when $\alpha=1$.

Results from Tables 7- 8 suggest that the two-stage stochastic model can lead to significant 


\begin{tabular}{|c|c|c|c|c|c|c|c|}
\hline Combin. & Version & T.time(s.) & T.timeEV(s.) & $V S S_{F}$ & $V S S_{D}$ & $V S S_{E}$ & $V S S_{T}$ \\
\hline \multirow{5}{*}{ U/Den./R } & 0 & 28.57 & 27.74 & $3.66 \%$ & $-1.17 \%$ & $9.86 \%$ & $12.34 \%$ \\
\hline & 1 & 186.96 & 514.57 & $2.90 \%$ & $-2.11 \%$ & $12.48 \%$ & $13.26 \%$ \\
\hline & 2 & 885.44 & $6,448.55$ & $0.95 \%$ & $-2.18 \%$ & $14.83 \%$ & $13.59 \%$ \\
\hline & 3 & $3,618.79$ & $7,142.61$ & $2.37 \%$ & $-1.85 \%$ & $14.02 \%$ & $14.54 \%$ \\
\hline & 4 & $7,133.13$ & $7,125.84$ & - & - & - & - \\
\hline \multirow{5}{*}{ B/Den./R } & 0 & 123.44 & $1,205.76$ & $1.98 \%$ & $-3.45 \%$ & $15.16 \%$ & $13.69 \%$ \\
\hline & 1 & 573.15 & $5,451.17$ & $-0.29 \%$ & $-3.03 \%$ & $16.45 \%$ & $13.13 \%$ \\
\hline & 2 & $3,790.71$ & $7,032.83$ & $0.71 \%$ & $-4.35 \%$ & $17.47 \%$ & $13.83 \%$ \\
\hline & 3 & $5,659.21$ & $7,162.05$ & - & - & - & - \\
\hline & 4 & $6,862.59$ & $7,174.46$ & - & - & - & - \\
\hline \multirow{5}{*}{ U/Dis./NR } & 0 & 14.45 & 5.89 & $-0.72 \%$ & $2.97 \%$ & $1.12 \%$ & $3.36 \%$ \\
\hline & 1 & 128.15 & 52.11 & $-0.31 \%$ & $2.23 \%$ & $1.58 \%$ & $3.50 \%$ \\
\hline & 2 & 437.86 & 188.91 & $0.29 \%$ & $1.84 \%$ & $1.39 \%$ & $3.52 \%$ \\
\hline & 3 & $1,047.65$ & 471.71 & $-0.07 \%$ & $2.07 \%$ & $1.66 \%$ & $3.65 \%$ \\
\hline & 4 & $3,213.85$ & 998.59 & $-0.28 \%$ & $1.85 \%$ & $1.28 \%$ & $2.85 \%$ \\
\hline \multirow{5}{*}{ U/Den./NR } & 0 & 16.09 & 4.82 & $-2.14 \%$ & $0.96 \%$ & $15.21 \%$ & $14.03 \%$ \\
\hline & 1 & 530.62 & 134.19 & $-3.31 \%$ & $0.19 \%$ & $17.95 \%$ & $14.83 \%$ \\
\hline & 2 & $1,776.50$ & $5,647.53$ & $-4.43 \%$ & $1.82 \%$ & $18.59 \%$ & $15.99 \%$ \\
\hline & 3 & $5,215.26$ & $6,412.88$ & $-3.68 \%$ & $1.09 \%$ & $17.89 \%$ & $15.30 \%$ \\
\hline & 4 & $5,600.60$ & $6,603.05$ & $-4.46 \%$ & $1.17 \%$ & $18.47 \%$ & $15.18 \%$ \\
\hline \multirow{5}{*}{ B/Dis./NR } & 0 & 51.18 & 33.50 & $6.90 \%$ & $0.80 \%$ & $-6.79 \%$ & $0.90 \%$ \\
\hline & 1 & 574.56 & 427.75 & $5.46 \%$ & $0.73 \%$ & $-5.04 \%$ & $1.15 \%$ \\
\hline & 2 & $1,079.79$ & $2,492.02$ & $2.13 \%$ & $0.03 \%$ & $-1.23 \%$ & $0.93 \%$ \\
\hline & 3 & $3,247.74$ & $3,369.72$ & $6.06 \%$ & $0.25 \%$ & $-4.90 \%$ & $1.41 \%$ \\
\hline & 4 & $4,789.92$ & $6,553.39$ & $8.30 \%$ & $-0.14 \%$ & $-7.89 \%$ & $0.28 \%$ \\
\hline \multirow{5}{*}{ B/Den./NR } & 0 & 48.71 & 542.38 & $-0.30 \%$ & $-0.48 \%$ & $10.21 \%$ & $9.43 \%$ \\
\hline & 1 & 485.00 & $5,087.42$ & $1.21 \%$ & $-1.51 \%$ & $10.66 \%$ & $10.36 \%$ \\
\hline & 2 & $4,268.23$ & $6,467.77$ & $1.62 \%$ & $-1.54 \%$ & $10.21 \%$ & $10.29 \%$ \\
\hline & 3 & $6,235.23$ & $6,635.15$ & $8.34 \%$ & $-4.44 \%$ & $4.81 \%$ & $8.70 \%$ \\
\hline & 4 & $7,200.41$ & $6,779.29$ & - & - & - & - \\
\hline
\end{tabular}

Table 8 - Value of the stochastic solution when $\alpha=2$.

reductions in costs when compared to the mean value program, since most of the $V S S_{T}$ are positive values ranging from $1.21 \%$ to $16.22 \%$ when $\alpha=1$, and ranging from $0.28 \%$ to $15.99 \%$ when $\alpha=2$. We observe that the expected reduction or the expected increase in the three types of costs (shift allocation costs, order allocation costs for the dedicated couriers, and external courier order allocation costs) by using the stochastic model is influenced by the spatial distribution of new requests ( $R$ and $N R$ ) and by the density of demand (Dis. and Den.). For instance, when $\alpha$ is equal to one and the demand is dense, we observe that there is a large decrease in the costs of external couriers; cost that is compensated by an increase in the order allocation costs to dedicated couriers and by an increase in the shift allocation costs (some $V S S_{D}$ and $V S S_{F}$ take negative values). On the contrary, when $\alpha$ is equal to one and the demand is disperse, we notice that there is an increase or just a small change the external cost, and that there is a decrease in the the order allocation costs to dedicated couriers and in the shift allocation costs, as the values for $V S S_{F}, V S S_{D}$ are positive. The values for $V S S_{F}, V S S_{D}$, and $V S S_{E}$ when $\alpha=2$ show a similar behavior than the values for $V S S_{F}, V S S_{D}$, and $V S S_{E}$ when $\alpha=1$. However, as it can be seen in Table 8 , an increase in the value of $\alpha$ generally increases (in a small amount) the total value of the stochastic solution $\left(V S S_{T}\right)$ for the instances with random spatial distribution. On the contrary, the total value of the stochastic solution shows a small decrease when $\alpha$ changes from one to two for the instances with a non-random spatial distribution.

When $\alpha=1$, for most of the instances evaluated, we observe that the total CPU time to solve the stochastic problem is larger than the total CPU time to solve the deterministic problem. However, 
when $\alpha=2$ we note that there is no significant difference between the total CPU time to solve these two problems for most of the instances. This might be explained by the fact that the multi-cut L-shaped method provides more cuts (as the method adds one cut per time period and per scenario) to support the recourse function from below, and most likely reduce the number of iterations.

Last, it is noteworthy to mention that the value and importance of $V S S_{F}, V S S_{D}$, and $V S S_{E}$ might depend on the value for the fixed costs and variable costs, as well as on the probability distribution of same-day client orders. For instance, if the order delivery costs associated with the external couriers are significantly higher than the order delivery costs related to the dedicated couriers, an increase in the dedicated courier working hours (longer shifts) by using the stochastic model will lead to a large percentage improvement in the external cost (larger value for $V S S_{E}$ ).

\subsection{Evaluation of the Incorporation of the Tactical Planning Solutions into the Operational Planning}

In this Section, we evaluate the impact on the incorporation of tactical planning solutions into the operational planning. To that end, we solve the operational planning problem under two scenarios. First, as described in Section 4 , we include the tactical planning solutions obtained when $\alpha=2$ to build sets $C_{t}$ and $O_{t}^{c}$. Second, we assume that couriers have not been assigned to any working shift and that o-d pairs have not been allocated to them. Therefore, the initial filter to build set $C_{t}$ only includes the couriers availabilities and their preferences, and set $O_{t}^{c}$ is composed by including the already assigned requests to courier $c$ at instant $t$, as well as the new orders $O_{t}^{N}$ arriving at $t$. Table 9 presents the results of this evaluation for the instances with combination U/Den./R, U/Dis./NR, and U/Den./NR. Columns labeled as Cost $_{F}$, Cost $_{D}$, Cost $_{E}$, and Cost $_{T}$ present the differences between the shift allocation costs, the order allocation cost to dedicated couriers, the order allocation costs to external couriers, and the total costs, respectively, when the operational planning problem is solved for the two scenarios. The differences between the number of dedicated couriers used, between the average number of packages assigned per courier, and between the dispersion in the working hours are presented in the columns labeled as Couriers, Pack./courier, and Dispersion, respectively. The Dispersion is computed as the maximum number of hours worked among all couriers. The differences in costs are computed as: Cost $_{c}=100 \times\left(\right.$ Cost $_{c}^{\prime}-$ Cost $\left._{c}^{T}\right) /$ Cost $_{c}^{\prime}$, for all $c=\{F, D, E, T\}$, where $\operatorname{Cost}_{c}^{T}$ and Cost $_{c}^{\prime}$ correspond to the value for the costs when the tactical solutions are incorporated into the operational planning, and to the value for the costs when the tactical solutions are not considered, respectively. The values for Couriers, Pack./courier, and Dispersion are computed in the same way as for Cost $_{c}$.

\begin{tabular}{ccccccccc}
\hline Combin. & Version & Cost $_{F}$ & Cost $_{D}$ & Cost $_{E}$ & Cost $_{T}$ & Couriers & Pack./courier & Dispersion \\
\hline \multirow{3}{*}{ U/Den./R } & 1 & $77.29 \%$ & $55.22 \%$ & $-96.16 \%$ & $-11.62 \%$ & $28.5 \%$ & $1.06 \%$ & $25.6 \%$ \\
& 2 & $115.35 \%$ & $15.12 \%$ & $-97.11 \%$ & $-7.65 \%$ & $52.26 \%$ & $-19.95 \%$ & $26.95 \%$ \\
& 3 & $112.16 \%$ & $4.19 \%$ & $-98.69 \%$ & $-8.91 \%$ & $63.55 \%$ & $-27.62 \%$ & $31.11 \%$ \\
\hline \multirow{3}{*}{ U/Dis./NR } & 1 & $-12.98 \%$ & $66.61 \%$ & $-95.06 \%$ & $-10.3 \%$ & $-6.61 \%$ & $24.5 \%$ & $28.1 \%$ \\
& 2 & $-1.45 \%$ & $38.16 \%$ & $-98.62 \%$ & $-7.72 \%$ & $-4.83 \%$ & $15.27 \%$ & $29.29 \%$ \\
& 3 & $-1.06 \%$ & $32.28 \%$ & $-94.19 \%$ & $-4.72 \%$ & $-6.17 \%$ & $14.96 \%$ & $37.49 \%$ \\
\hline \multirow{3}{*}{ U/Den./NR } & 1 & $9.74 \%$ & $55.76 \%$ & $-93.33 \%$ & $-7.8 \%$ & $-6.67 \%$ & $25.6 \%$ & $29.65 \%$ \\
& 2 & $62.64 \%$ & $24.39 \%$ & $-97.09 \%$ & $-5.35 \%$ & $42.61 \%$ & $-18.08 \%$ & $27.35 \%$ \\
& 3 & $83.86 \%$ & $9.92 \%$ & $-98.77 \%$ & $-5.76 \%$ & $42.54 \%$ & $-19.24 \%$ & $28.62 \%$ \\
\hline
\end{tabular}

Table 9 - Operational planning results to evaluate the incorporation of the stochastic tactical solutions.

Results from Table 9, indicate that the total cost $\left(\right.$ Cost $\left._{T}\right)$ and the external courier cost $\left(\right.$ Cost $\left._{E}\right)$ 
show a reduction when tactical solutions are not incorporated into the operational planning, as the operational model has the freedom to assign each order to all dedicated couriers, at any time of the day, in any geographic zone. However, these results also show that dedicated couriers tend to be underutilized when tactical solutions are not included into the operational planning, as sometimes they are assigned to less packages (see negative values for Pack./courier), over longer workdays (see positive values for Dispersion). It is noteworthy to mention that more than achieving a reduction in the total costs, Colisweb is interested into having a solution approach to generate solutions that will assign balanced workloads to the couriers, in order to improve their satisfaction and the client's service level. This balance is measured by three aspects: the number of working hours, the number of orders to deliver at each working hour, and the number of different geographic zones to visit at each working time period. The incorporation of the tactical solutions generated by the two-stage stochastic problem, helps to improve these three aspects increasing the couriers' working efficiency and helping the couriers to become familiarized with the personnel working at the depots, as well as with the final clients.

Table 10 presents a comparison between the operational costs obtained when the tactical solutions resulting from the two-stage stochastic problem and when the tactical solutions resulting from the mean value problem are incorporated into the operational planning. Specifically, we present the difference for the shift allocation costs $\left(\right.$ Cost $\left._{F}\right)$, the difference for the dedicated courier order allocation costs $\left(\operatorname{Cost}_{D}\right)$, the difference for the external courier order allocation costs $\left(\operatorname{Cost}_{E}\right)$, and the different for the total costs $\left(\operatorname{Cost}_{T}\right)$. These values are computed as: $\operatorname{Cost}_{c}=100 \times\left(E E V_{c}-R P_{c}\right) / E E V_{c}$, for all $c=\{F, D, E, T\}$, where $E E V_{F}, E E V_{D}, E E V_{E}$, and $E E V_{T}$ correspond to the values for the shift allocation cost, for the dedicated courier order allocation cost, for the external courier order allocation cost, and for the total cost, when using, in the operational planning, the tactical solutions found with the mean value problem. In the same way, $R P_{F}, R P_{D}, R P_{E}$, and $R P_{T}$ correspond to the final values for the shift allocation cost, for the dedicated courier order allocation cost, for the external courier order allocation cost, and for the total cost when using, in the operational planning, the tactical solutions found with the two-stage stochastic model.

\begin{tabular}{cccccc}
\hline Combin. & Version & Cost $_{F}$ & Cost $_{D}$ & Cost $_{E}$ & Cost $_{T}$ \\
\hline \multirow{3}{*}{ U/Den./R } & 1 & $17.74 \%$ & $-1.92 \%$ & $-0.66 \%$ & $3.98 \%$ \\
& 2 & $18.13 \%$ & $-2.67 \%$ & $13.1 \%$ & $6.16 \%$ \\
& 3 & $5.7 \%$ & $2.39 \%$ & $14.18 \%$ & $6.33 \%$ \\
\hline \multirow{3}{*}{ U/Dis./NR } & 1 & $-1.54 \%$ & $1.94 \%$ & $1.5 \%$ & $1.19 \%$ \\
& 2 & $-0.71 \%$ & $1.95 \%$ & $3.73 \%$ & $1.79 \%$ \\
& 3 & $0.59 \%$ & $3.24 \%$ & $9.32 \%$ & $3.22 \%$ \\
\hline \multirow{3}{*}{ U/Den./NR } & 1 & $-6.67 \%$ & $6.65 \%$ & $17.34 \%$ & $6.53 \%$ \\
& 2 & $-13.67 \%$ & $10.57 \%$ & $23.27 \%$ & $10.13 \%$ \\
& 3 & $-16.78 \%$ & $10.43 \%$ & $17.89 \%$ & $8.61 \%$ \\
\hline
\end{tabular}

Table 10 - Comparison of the operational costs when using the stochastic tactical solutions and the mean value tactical solutions.

The values from Table 10 can be interpreted as the value of the stochastic solution $(V S S)$ when the tactical solutions are incorporated into the operational planning. These results indicate that stochastic tactical solutions are more robust than the mean value tactical solutions, as all Cost $_{T}$ are positive values ranging from $1.19 \%$ to $10.13 \%$. Observe that, the operational planning under the stochastic tactical solutions presents a better reaction to fluctuations in client orders than the operational planning under the mean value tactical solutions, as the cost of using external couriers, Cost $_{E}$, is positive for almost all the instances evaluated. 


\section{Concluding Remarks}

This paper proposed a two-stage stochastic programming model to solve an integrated shift scheduling and load assignment problem for attended home delivery with uncertain order requests. In the model, first-stage decisions correspond to the allocation of couriers to shifts and to geographic zones at each working time period. Second-stage decisions correspond to the allocation of client orders to couriers (dedicated and external).

A multi-cut L-shaped method was implemented as a solution approach. Some algorithmic refinements were used to enhance the performance of the method. First, we adopted the strategy of McDaniel and Devine (1977) to generate an initial set of valid cuts in a fast way. Second, we derive some valid inequalities and develop a special case of the problem to improve the lower bound and to accelerate the convergence of the method, respectively.

An extensive computational study suggests that our algorithm is able to provide solutions with a maximum average optimality gap of $1.07 \%$ for instances dealing with up to 150 client orders, 42 couriers, 23 scenarios, and a maximum of 2 origin destination pairs to visit per courier, per time period $(\alpha=2)$. For the larger instances, results show that good-quality solutions can be achieved if the number of geographic zones that each courier can visit at each time period is set to one, i.e., $\alpha=1$. The value of the stochastic solution, ranging from $1.21 \%$ to $16.22 \%$ when $\alpha=1$ and from $0.28 \%$ to $15.99 \%$ when $\alpha=2$, demonstrates that the two-stage model leads to tactical solutions achieving significant reductions in costs when compared to the mean value program, since it prevents the occurrence of additional external and courier underutilization costs. The robustness of the tactical solutions was evaluated by incorporating the working shift assignment and geographic zone allocation into the operational planning. Results show that this incorporation leads to a better distribution of the working time and order allocation to dedicated couriers, improving the couriers' working efficiency and thus the customers' service level.

An interesting avenue for future research is related to a more precise estimation of the travel and service times needed by each courier, in order to deliver the packages in a specific time period and geographic zone (o-d pair). Future research can also include the definition of the tactical problem over longer time horizons, i.e. generation of weekly courier schedules instead of daily shifts, as well as the development of hybrid methods (e.g., hybrid between heuristics and exact methods) to tackle larger instances.

\section{References}

Agatz N, Campbell AM, Fleischmann M, Savelsbergh M, 2008 Challenges and opportunities in attended home delivery. The vehicle routing problem: latest advances and new challenges, 379-396 (Springer).

Agatz N, Campbell AM, Fleischmann M, Savelsbergh M, 2011 Time slot management in attended home delivery. Transportation Science 45(3):435-449.

Bent RW, Van Hentenryck P, 2004 Scenario-based planning for partially dynamic vehicle routing with stochastic customers. Operations Research 52(6):977-987.

Birge JR, Louveaux FV, 1988 A multicut algorithm for two-stage stochastic linear programs. European Journal of Operational Research 34(3):384-392.

Campbell AM, Savelsbergh M, 2005 Decision support for consumer direct grocery initiatives. Transportation Science 39(3):313-327.

Campbell AM, Savelsbergh M, 2006 Incentive schemes for attended home delivery services. Transportation science 40(3):327-341.

Ehmke JF, Campbell AM, 2014 Customer acceptance mechanisms for home deliveries in metropolitan areas. European Journal of Operational Research 233(1):193-207. 
Hernandez F, Gendreau M, Potvin JY, 2014 Heuristics for time slot management: A periodic vehicle routing problem view. Technical Report 59, CIRRELT.

Klein R, Neugebauer M, Ratkovitch D, Steinhardt C, 2016 Differentiated time slot pricing under routing considerations in attended home delivery. Available at SSRN 2674061.

Laporte G, Louveaux FV, 1993 The integer L-shaped method for stochastic integer programs with complete recourse. Operations research letters 13(3):133-142.

M-M S, Krishnamurti R, Laporte G, 2004 Double-horizon based heuristics for the dynamic pickup and delivery problem with time windows. Transportation Research Part B: Methodological 38(8):669-685.

McDaniel D, Devine M, 1977 A modified Benders' partitioning algorithm for mixed integer programming. Management Science 24(3):312-319.

Pillac V, Gendreau M, Guéret C, Medaglia AL, 2013 A review of dynamic vehicle routing problems. European Journal of Operational Research 225(1):1-11.

Ritzinger U, Puchinger J, Hartl RF, 2016 A survey on dynamic and stochastic vehicle routing problems. International Journal of Production Research 54(1):1-17.

Sungur I, Ren Y, Ordóñez F, Dessouky M, Zhong H, 2010 A model and algorithm for the courier delivery problem with uncertainty. Transportation Science 44(2):193-205.

Ulmer MW, Mattfeld DC, Hennig M, Goodson JC, 2016 Logistics Management: Contributions of the Section Logistics of the German Academic Association for Business Research, 2015, Braunschweig, Germany, chapter A Rollout Algorithm for Vehicle Routing with Stochastic Customer Requests, 217-227 (Springer International Publishing), URL http://dx.doi.org/10.1007/978-3-319-20863-3_16.

Ulmer MW, Mattfeld DC, Köster F, 2014 Anticipation of stochastic customer requests in vehicle routing: Value function approximation based on a dynamic lookup-table. Technical report, working paper.

Van Slyke RM, Wets R, 1969 L-shaped linear programs with applications to optimal control and stochastic programming. SIAM Journal on Applied Mathematics 17(4):638-663.

Wolsey LA, Nemhauser GL, 1999 Integer and combinatorial optimization (John Wiley \& Sons).

Yan S, Lin JR, Lai CW, 2013 The planning and real-time adjustment of courier routing and scheduling under stochastic travel times and demands. Transportation Research Part E: Logistics and Transportation Review 53:34-48.

Yang X, Strauss AK, Currie CS, Eglese R, 2016 Choice-based demand management and vehicle routing in e-fulfillment. Transportation science 50(2):473-488. 


\section{Appendix A Constraint Lifting}

Let $z_{i p}^{c *}$ be the optimal value of problem (42)- 44 when o-d pair $p$ is already assigned to courier $c$ at time period $i$. The optimal value $z_{i p}^{c *}+1$ represents the maximum number of o-d pairs that can be visited per courier $c$ at time period $i$, if only the total distance constraints 43 are considered.

$$
\begin{aligned}
\max z_{i p}^{c}= & \sum_{p^{\prime} \in P \mid p^{\prime} \neq p} y_{i p^{\prime}}^{c} \\
& \sum_{\substack{p^{\prime} \in P\left|p^{\prime} \neq p \\
y_{i p^{\prime}}^{c} \in\{0,1\}, \forall p^{\prime} \in P\right| p^{\prime} \neq p .}} \sigma_{i p^{\prime}}^{c} y_{i}^{c} \leq \tau_{i}^{c}-\sigma_{i p}^{c},
\end{aligned}
$$

The lifted value for the maximum number of o-d pairs to cover at each time period $i$ by each courier $c, \alpha_{i}^{c *}$, is determined by equation (45). Equation (46) gives the lifted coefficient of variable $y_{i p}^{c}$, in constraint (3) from model ISSLA.

$$
\begin{array}{r}
\alpha_{i}^{c *}=\min \left\{\alpha_{i}^{c}, \max _{p^{\prime} \in P}\left\{z_{i p}^{c *}+1\right\}\right\}, \\
\chi_{i p}^{c}= \begin{cases}\alpha_{i}^{c *}-z_{i p}^{c *} & \text { if } z_{i p}^{c *}<\alpha_{i}^{c *}-1 \\
1 & \text { otherwise }\end{cases}
\end{array}
$$

\section{Appendix B SSLA Model Reformulation when $\alpha_{\mathbf{i}}^{\mathbf{c}}=1, \forall \mathbf{c} \in \mathbf{C}$ for at least one $\mathbf{i} \in \mathbf{I}$}

Let $I_{1}, I_{2}$ be a division of set $I$ such that $I=I_{1} \cup I_{2}$. $I_{1}$ represents the set of time periods in which all couriers must visit at most one o-d pair (i.e., $\alpha_{i}^{c}=1, \forall c \in C, i \in I_{1}$ ). On the contrary, $I_{2}$ represents the set of time periods in which at least one courier can visit more than one o-d pair (i.e., $\alpha_{i}^{c}>1, \forall c \in C, i \in I_{2}$ ). When $\alpha_{i}^{c}=1$ for every $c \in C$ at a given time period $i \in I_{1}$, the resulting second-stage problem (13)-(18) for time period $i$ and scenario $w_{i}$ can be decomposed into $|P|$ independent sorting problems, denoted by $\mathcal{Q}\left(\mathbf{y}_{i p}, \xi_{i}(w)\right)$ and the two-stage stochastic problem (1)-(11) can be reformulated as: 


$$
\begin{aligned}
& \min \sum_{c \in C} \sum_{s \in S^{c}} f_{s}^{c} x_{s}^{c}+\sum_{i \in I_{1}} \sum_{w \in \Omega_{i}} \sum_{p \in P} \theta_{i p}^{(w)}+\sum_{i \in I_{2}} \sum_{w \in \Omega_{i}} \theta_{i}^{(w)} \\
& \sum_{s \in S^{c}} x_{s}^{c} \leq 1, \forall c \in C, \\
& \sum_{p \in P} y_{i p}^{c}=\sum_{s \in S^{c}} \delta_{i s}^{c} x_{s}^{c}, \forall c \in C, i \in I_{1}, \\
& \sum_{s \in S^{c}} \delta_{i s}^{c} x_{s}^{c} \leq \sum_{p \in P} y_{i p}^{c} \leq \alpha_{i}^{c} \sum_{s \in S^{c}} \delta_{i s}^{c} x_{s}^{c}, \forall c \in C, i \in I_{2}, \\
& \sum_{p \in P} \sigma_{i p}^{c} y_{i p}^{c} \leq \tau^{c} \sum_{s \in S^{c}} \delta_{i s}^{c} x_{s}^{c}, \forall c \in C, i \in I, \\
& \theta_{i p}^{(w)} \geq p_{i}^{(w)}\left(\sum_{c \in C} \mu_{i p}^{c} \zeta_{i p}^{c(w)} y_{i p}^{c}+d_{i p}^{(w)} \eta_{i p}^{(w)}\right) \\
& \forall i \in I_{1}, w \in \Omega_{i}, p \in P,\left(\pi_{i p}^{(w)}, \lambda_{i p}^{(w)}\right) \in E_{\boldsymbol{\Delta}_{i p}^{(w)}}, \\
& \theta_{i}^{(w)} \geq p_{i}^{(w)}\left(\sum_{c \in C} \sum_{p \in P} \mu_{i p}^{c} \pi_{i p}^{c(w)} y_{i p}^{c}+\sum_{c \in C} \beta_{i}^{c} \phi_{i}^{c(w)} \sum_{s \in S^{c}} \delta_{i s}^{c} x_{s}^{c}+\sum_{p \in P} d_{i p} \lambda_{i p}^{(w)}\right), \\
& \forall i \in I_{2}, w \in \Omega_{i},\left(\pi_{i}^{(w)}, \phi_{i}^{(w)}, \lambda_{i}^{(w)}\right) \in E_{\boldsymbol{\Delta}_{i}^{(w)}}, \\
& \sum_{p^{\prime} \notin N_{p}} y_{i p^{\prime}}^{c} \leq 1-y_{i p}^{c}, \forall c \in C, i \in I, p \in P, \\
& z_{i p}^{c} \leq y_{i p}^{c}, \forall c \in C, i \in I, p \in P, \\
& \sum_{p \in P} z_{i p}^{c} \geq \sum_{s \in S^{c}} \delta_{i s}^{c} x_{s}^{c}, \forall c \in C, i \in I, \\
& z_{i p}^{c}+\sum_{s \in S^{c}} \delta_{i+1 s}^{c} x_{s}^{c} \leq \sum_{p^{\prime} \in N_{p}} y_{i+1 p^{\prime}}^{c}+1, \forall c \in C, i \in I, p \in P, \\
& x_{s}^{c} \geq 0, \forall c \in C, s \in S^{c}, \\
& y_{i p}^{c} \in\{0,1\}, \forall c \in C, i \in I, p \in P \text {, } \\
& z_{i p}^{c} \in\{0,1\}, \forall c \in C, i \in I, p \in P \text {, } \\
& \theta_{i p}^{(w)} \geq 0, \forall i \in I_{1}, w \in \Omega_{i}, p \in P, \\
& \theta_{i}^{(w)} \geq 0, \forall i \in I_{2}, w \in \Omega_{i} \text {. }
\end{aligned}
$$

Where $\zeta_{i p}^{c(w)}, \eta_{i p}^{(w)}$ are the values of the dual variables associated with the dual of the second-stage problem 63- 67) presented below, and $\boldsymbol{\Delta}_{i p}^{(w)}$ is the projection over the space of variables $\zeta_{i p}^{c(w)}, \eta_{i p}^{(w)}$ of the polyhedron defined by the constraints associated with the dual of (63)-(67). 


$$
\begin{aligned}
\mathcal{Q}\left(\mathbf{y}_{i p}, \xi_{i}(w)\right)=\min & \sum_{c \in C} l_{i p}^{c} v_{i p}^{c(w)}+c_{i p} e_{i p}^{(w)} \\
& v_{i p}^{c(w)} \leq \mu_{i p}^{c(w)} y_{i p}^{c}, \forall c \in C, \\
& \sum_{c \in C} v_{i p}^{c(w)}+e_{i p}^{(w)}=d_{i p}^{(w)}, \\
& v_{i p}^{c(w)} \geq 0, \forall c \in C, \\
& e_{i p}^{(w)} \geq 0 .
\end{aligned}
$$

